\title{
Comparison of REV size and tensor characteristics for the electrical and hydraulic conductivities in fractured rock
}

\author{
Serdar Demirel $^{1}$, James Irving ${ }^{1 *}$, and Delphine Roubinet ${ }^{2}$ \\ ${ }^{1}$ Institute of Earth Sciences, University of Lausanne, Switzerland \\ ${ }^{2}$ Geosciences Montpellier (CNRS), University of Montpellier, France \\ * Corresponding author (james.irving@unil.ch) \\ Revised draft for Geophysical Journal International \\ Abbreviated title: Comparison of REV size and tensor characteristics
}

\section{Summary}

\section{Th} roc

a p conductivity $(K)$ in fractured rock have been the subject of numerous previous investigations, no studies to date have focused on the electrical conductivity $(\sigma)$. This is despite the fact that geoelectrical measurements are arguably the most popular means of geophysically investigating fractured rock, typically via azimuthal resistivity surveying where the observed electrical anisotropy is commonly used to infer hydraulic characteristics. In this paper, we attempt to fill this void and present a systematic numerical study of the impacts of changes in fracture-network properties on the REV size and equivalent tensor characteristics for both the electrical and hydraulic conductivities. We employ a combined statistical and numerical approach where the size of the REV is estimated from the conductivity variability observed across multiple stochastic fracture-network realizations for various domain sizes. Two important differences between fluid and electric current flow in fractured media are found to 
lead to significant differences in the REV size and tensor characteristics for $\sigma$ and $K$; these are the greater importance of the matrix in the electrical case and the single-power instead of cubic dependence of electric current flow upon aperture. Specifically, the REV for the electrical conductivity will always be smaller than that for the hydraulic conductivity, and the corresponding equivalent tensor will exhibit less anisotropy, often with notably different principal orientations. These findings are of key importance for the eventual interpretation of geoelectrical measurements in fractured rock, where we conclude that extreme caution must be taken when attempting to make the link to hydraulic properties.

Keywords: electrical conductivity; hydraulic conductivity; representative elementary volume (REV); representative volume element (RVE); tensor; discrete-dual-porosity (DDP); discrete fracture network (DFN); resistivity; stochastic; geoelectrical

\section{Introduction}

Fractured rocks play a critically important role in a wide variety of geoscience problems including groundwater flow and contaminant transport, aquifer remediation, hydrocarbon extraction, geothermal resource exploitation, and the long-term underground storage of $\mathrm{CO}_{2}$ and nuclear waste (Carneiro, 2009; Follin et al., 2014; Geiger \& Emmanuel, 2010; Kolditz \& Clauser, 1998; Manna et al., 2017; Neuman, 2005; Rotter et al., 2008; Zhao et al., 2017). Numerous studies have been devoted to the development of methods for the identification and characterization of subsurface fractures and fracture networks, with the overall aim of improving conceptual and numerical models of flow and transport in fractured-rock environments (Berkowitz, 2002; Bonnet et al., 2001; Davy et al., 2010; NAP, 2015). In this regard, applied geophysical methods have gained widespread interest, as many of these methods are highly sensitive to the presence of fractures and the corresponding measurements can be acquired quickly and non-invasively from the Earth's surface and/or from boreholes. Examples include ground-penetrating radar (Dorn et al., 2012; Tsoflias et al., 2001), seismic (Herwanger et al., 2004a; Pytharouli et al., 2011), electrical resistivity (Lane et al., 1995; Robert et al., 2012), induced polarization (Marescot et al., 2008; Schmutz et al., 2011), self potential 
(DesRoches et al., 2017; Roubinet et al., 2016; Wishart et al., 2008), and electromagnetic methods (Donadille \& Al-Ofi, 2012; Steelman et al., 2015).

Amongst the multitude of geophysical techniques that have been applied to fractured-rock problems, the electrical resistivity (ER) method is of particular interest because: (i) numerous field, laboratory, and theoretical studies have demonstrated that geoelectrical data are significantly affected by the presence and characteristics of fractures such as density, orientation, and aperture (Jinsong et al., 2009; Taylor \& Fleming, 1988); (ii) ER measurements can be acquired using a variety of electrode configurations and spacings, thereby offering the potential to obtain information on subsurface properties over a wide range of spatial scales (Everett, 2013; Telford et al., 1990); and (iii) strong analogies between fluid and electric current flow in fractured media suggest that geoelectrical data may contain important information regarding the corresponding hydrogeological properties (Brown, 1989; Van Siclen, 2002). As a result, ER studies in fractured rock have been widespread, and include the development and application of anisotropic tomographic methods (Greenhalgh et al., 2009; Herwanger et al., 2004a,b; Li \& Spitzer, 2005; Pain et al., 2003); the use of azimuthal resistivity surveys to estimate predominant fracture orientations (Al Hagrey, 1994; Busby, 2000; Lane et al., 1995; Taylor \& Fleming, 1988) along with, in many cases, properties of the hydraulic conductivity tensor (Boadu et al., 2005, 2008; Ritzi \& Andolsek, 1992; Skinner \& Heinson, 2004; Skjernaa \& Jørgensen, 1994; Steinich \& Marin, 1996; Yeboah-Forson \& Whitman, 2014); and the acquisition of surface-based electrical resistivity tomography (ERT) profiles for the identification and characterization of fracture zones (Porsani et al., 2005; Robert et al., 2011, 2012; Sharma \& Baranwal, 2005; Yadav \& Singh, 2007).

One concept that is absolutely critical when it comes to making meaningful use of geoelectrical data in fractured-rock investigations is the representative elementary volume (REV). In materials engineering, the REV is typically defined as the minimum volume of heterogeneous material that is large enough to be statistically representative of the composite with respect to a particular physical property (Kanit et al., 2003). In other words, at volume sizes greater than the REV, small-scale heterogeneities in the medium need not be explicitly taken into account because their effects can be adequately captured by a set of average continuum 
properties. With regard to geoelectrical measurements in fractured rock, the REV defines the scale beyond which the electrical conductivity of the fractured medium can be adequately described using a second-order tensor, thus avoiding the need to consider the detailed effects of individual fractures on the passage of electric current. Knowledge about the REV is essential for understanding under what circumstances ERT forward modeling and inversion codes, which are based upon a discretized parameterization of the conductivity, can be effectively utilized. Indeed, such codes inherently assume that the chosen model-cell size is at or beyond the scale at which conductivity can be effectively described by a scalar or tensor, which may or may not be valid. The notion of REV is also fundamental for understanding when and how bulk electrical properties can be related to those of the underlying fracture network (i.e., fracture densities, orientations, lengths, and apertures). Finally, it is essential to understand the nature of the REV for the electrical conductivity in fractured rock, and its relationship to the REV for the hydraulic conductivity, before conclusions can be made about subsurface hydrogeological properties based on geoelectrical measurements. As noted above, a number of researchers have taken the step of linking the results of azimuthal resistivity surveying to properties of the hydraulic conductivity tensor, with the justification that fluid and electric current will take similar paths through the fractured medium based on parallels between Ohm's and Darcy's laws. No study to date, however, has ever critically examined the validity of this approach in the context of realistic fracture networks.

Within the hydrogeological community, the existence and properties of the REV for the hydraulic conductivity in fractured rock have been rather extensively investigated. Snow (1969) conducted an analytical study in which he derived the anisotropic permeability tensor for fractured media assuming sets of infinitely long parallel fractures having different orientations, apertures, and spacings. Using a 2D discrete-fracture-network (DFN) numerical modeling approach and assuming an impermeable matrix, Long et al. (1982) subsequently investigated the REV size and permeability tensor characteristics for a variety of fracture networks, where fracture positions, orientations, lengths, and apertures were drawn randomly from statistical distributions. More recent research has continued along these lines, examining through numerical DFN simulations how the REV size and permeability tensor are affected 
by the statistical distribution of fracture parameters (Min et al., 2004; Wang \& Kulatilake, 2008) as well as correlations between parameters (Baghbanan \& Jing, 2007). With respect to the electrical conductivity, on the other hand, there is a near complete lack of information in the literature on the existence and properties of an REV in fractured rock. While the effects of fractures on the equivalent conductivity beyond the REV scale have been examined for simple configurations (Berryman \& Hoversten, 2013; Jinsong et al., 2009), no studies have attempted to quantify the REV size and conductivity tensor characteristics for realistic fracture networks. The primary reason for this has been the absence of tools for numerically modeling electric current flow in fractured media. Whereas fluid flow can be rather easily examined because the rock matrix is often ignored on the basis that it is effectively impervious (Cvetkovic, 2017; Maillot et al., 2016; Neuman, 2005), this is not the case for the electrical conductivity where the matrix typically plays an important role in the conduction of electric current (Beskardes \& Weiss, 2018; Caballero Sanz et al., 2017; Roubinet \& Irving, 2014; Roubinet et al., 2016). As a result, modeling approaches that explicitly account for both the fractures and matrix, as well as interactions between these domains, are required. Unfortunately the use of standard numerical methods, for example finite-difference, -element, or -volume techniques where the fractures and matrix are fully discretized (Dey \& Morrison, 1979; Pidlisecky \& Knight, 2008; Rücker et al., 2006), has not been computationally feasible in this regard due to the extremely high number of model elements involved.

Recently, Roubinet \& Irving (2014) presented a novel numerical modeling approach for electric current flow in 2D fractured media that is based on a semi-analytical discrete-dualporosity formulation. For the first time, this methodology permits accurate computation of current flow through realistic and highly complex fracture networks with orders of magnitude less computational cost than standard numerical methods. Our goal in the current paper is to use this modeling approach to examine the REV size and tensor characteristics for the electrical conductivity in realistic fractured media, and to compare our findings with the corresponding results obtained for the hydraulic conductivity. This is done in full generality with respect to the $2 \mathrm{D}$ intrinsic equivalent medium properties, and not in the context of a particular field configuration or measurement setup. We examine the effects of changing fracture 
orientations, apertures, and lengths, as well as imposing statistical correlation between aperture and length. In Section 2 we present the overall methodology behind our approach, which involves (i) the stochastic generation of 2D square DFNs for various domain sizes; (ii) running fluid and electric current flow simulations; (iii) determination of the mean and variance of the estimated 2D conductivity tensor components as a function of domain size; and (iv) estimation of the average tensorial properties of the medium and the REV size. An advantage of the combined numerical and statistical approach to REV estimation considered in this paper is that numerical simulations need not be performed at the REV scale in order to estimate the REV size. We then show in Section 3 the results of applying this procedure to 16 different test cases, which allows us to draw conclusions about how the REVs for the electrical and hydraulic conductivity compare and are affected by changes in the fracture distribution. This leads to some general discussion regarding the validity of inferring characteristics of the hydraulic conductivity tensor from geoelectrical measurements, as well as implications for field measurements (Section 4).

\section{Methodology}

We use the combined numerical and statistical approach developed in Cailletaud et al. (1994) and Kanit et al. (2003) for our REV analysis, whereby the variance of the property of interest, quantified through the analysis of multiple stochastic realizations over several domain sizes, is used to establish a scaling relationship that permits definition of the REV in terms of a prescribed level of error. To this end, we generate random DFN realizations for different domain sizes based on chosen probability distributions for the fracture positions, orientations, lengths, and apertures. Numerical modeling of flow through the DFNs for two orthogonal sets of Dirichlet boundary conditions then allows estimation of the conductivity tensor components, whose mean values are used to determine the equivalent tensorial properties of the medium at and beyond the REV scale, and whose variability as a function of domain size is used to derive the scaling relationship required to estimate the size of the REV.

In accordance with previous work (Long et al., 1982; Min et al., 2004; Baghbanan \& Jing, 2007), we conduct our analysis in 2D in this paper. This has the strong advantages of 
(i) being orders of magnitude more computationally efficient than a 3D analysis while at the same time allowing for meaningful general conclusions to be made; and (ii) permitting use of the modeling framework of Roubinet \& Irving (2014) for the electric current flow problem, with no further developments required. Fractures in the 2D DFNs are represented as 1D linear elements having a constant aperture along their length, and are assumed to be filled with water. Although the latter is clearly a gross simplification of reality in the sense that is it well known that (i) aperture varies within fractures; (ii) fracture walls are rough; and (iii) fracture filling/alteration is common (Brown, 1989; Van Siclen, 2002), the aim of this study is to focus on the effects of the fracture-network rather than on details of the individual fractures. Indeed, all previous REV work has represented fractures using this simple parallelplate model. The various steps involved in our analysis are described in detail below.

\subsection{DFN generation}

Fractures in this study are completely described by their center position, orientation, length, and aperture, for which we define probablility distributions in order to generate a large number of stochastic DFN realizations. To create one of such realizations for a particular domain size, we populate an initial large-scale $(100 \times 100 \mathrm{~m})$ region with fractures, from which a central square sub-domain having the desired side length $L$ is extracted (Figure 1a and 1b). Fracture centers are assumed to be uniformly distributed in the $x$ and $y$ directions throughout the domain (Li et al., 2009; Li \& Zhang, 2010; Wang \& Kulatilake, 2008), meaning that the center point $\left(x_{c}, y_{c}\right)$ of each fracture can be obtained by drawing $x_{c}$ and $y_{c}$ from $\mathcal{U}[0,100]$. We consider a density of fracture centers of $2 \mathrm{~m}^{-2}$ for all of the examples considered in this paper, which is comparable with previous research efforts based on the analysis of fractures in the field (Baghbanan \& Jing, 2007; Min et al., 2004; Wang \& Kulatilake, 2008) and leads to the generation of 20,000 fractures in the $100 \times 100 \mathrm{~m}$ region. The latter parameter was not varied in our study as it is already well understood and rather intuitive that increasing the fracture density will tend to proportionally decrease the REV size and increase the overall magnitude of the domain conductivity (Li et al., 2009; Li \& Zhang, 2010; Long et al., 1982; Wang \& Kulatilake, 2008). Further, the fracture density value chosen in this paper is not 
expected to have an impact on the general findings and conclusions in Section 3.

[Figure 1 about here.]

Two fracture sets having different orientations are considered in each of the test cases examined in Section 3 (Li et al., 2009; Long et al., 1982; Wang \& Kulatilake, 2008). The fractures in the domain are distributed evenly between these two sets, and the orientation angles of fractures within each set are described by a normal distribution with mean $\mu_{\theta}$ and standard deviation $\sigma_{\theta}$. Although other statistical distributions have been considered to model fracture orientations in previous REV studies (Baghbanan \& Jing, 2007; Min et al., 2004), the normal distribution is the most common and straightforward choice (Li et al., 2009; Li \& Zhang, 2010; Long et al., 1982; Wang \& Kulatilake, 2008).

Fracture lengths are assumed to follow a power-law distribution, truncated at the lower end, whose probability density function (PDF) is given by

$$
f(\ell)= \begin{cases}k \ell^{-a} & \text { for } \ell \geq \ell_{\min } \\ 0 & \text { otherwise }\end{cases}
$$

where $\ell_{\min }$ is the minimum permitted fracture length, $a$ is the power-law exponent, and $k$ is a normalization constant that ensures that the PDF integrates to unity. Use of a power-law distribution is arguably the most common means of describing fracture lengths in recent literature (Baghbanan \& Jing, 2007; de Dreuzy et al., 2001; Min et al., 2004), and is supported by a substantial volume of work on the analysis of fracture traces observed at the Earth's surface as well as theoretical studies (Bonnet et al., 2001; Bour \& Davy, 1997; Davy et al., 2010). The latter research showed that the exponent $a$ typically ranges between 1 and 3 , with higher values corresponding to fracture networks dominated by short fractures and lower values describing networks where the connectivity is controlled by longer fractures. To generate random values for $\ell$ consistent with equation (1), we derive the associated cumulative distribution function (CDF) and use the inverse transform sampling approach (Devroye, 1986). 
The simulated value for the fracture length is obtained using

$$
\ell=\ell_{\min } X^{\frac{1}{1-a}},
$$

where $X$ is a uniform random number drawn from $\mathcal{U}[0,1]$.

Finally, fracture apertures in our study are assumed to be either constant within each fracture set (Min et al., 2004; Wang \& Kulatilake, 2008) or randomly distributed according to a truncated lognormal distribution (Baghbanan \& Jing, 2007; Li et al., 2009; Li \& Zhang, 2010; Long et al., 1982). The latter PDF is given by

$$
f(b)= \begin{cases}k \exp \left(-\frac{\left(\ln b-\mu_{\ln b}\right)^{2}}{2 \sigma_{\ln b}^{2}}\right) & \text { for } b_{\min } \leq b \leq b_{\max } \\ 0 & \text { otherwise }\end{cases}
$$

where $b_{\min }$ and $b_{\max }$ are the minimum and maximum permitted aperture values, $\mu_{\ln b}$ and $\sigma_{\ln b}$ are the mean and standard deviation of the natural logarithm of the aperture, and $k$ is again a normalization constant. As before, the inverse transform sampling approach can be used with the corresponding CDF to generate random aperture values according to equation (3). The simulated values are obtained using (Baghbanan \& Jing, 2007)

$$
b=\exp \left(\sqrt{2} \sigma_{\ln b} e^{-1}\left\{X \cdot\left(g\left(b_{\max }\right)-g\left(b_{\min }\right)\right)+g\left(b_{\min }\right)\right\}+\mu_{\ln b}\right)
$$

where $X$ is again a random number drawn from $\mathcal{U}[0,1], e^{-1}$ is the inverse error function, and $g(b)=\operatorname{erf}\left\{\left(\ln b-\mu_{\ln b}\right) / \sqrt{2} \sigma_{\ln b}\right\}$ with $\operatorname{erf}$ the error function. Note that a wide body of research indicates that fracture aperture tends to be positively correlated with length (Bonnet et al., 2001; Hatton et al., 1994; Klimczak et al., 2010; Neuman, 2008; Olson, 2003; Renshaw \& Park, 1997; Vermilye \& Scholz, 1995). To simulate values from the probability distributions in equations (1) and (3) while taking into account correlation between these variables, we simply use the same uniform random deviate $X$ to generate both values in equations (2) and (4), respectively (Baghbanan \& Jing, 2007). Also note that values for the parameters controlling the fracture aperture distribution were chosen in our study to yield apertures within the 
range of those "typically" encountered in fractured-rock environments (Lapcevic et al., 1997; Singhal \& Gupta, 2010).

\subsection{Fluid flow model}

To compute fluid flow through the different DFN realizations, we follow previous work and assume that the rock matrix can be effectively treated as impervious on the basis that its ability to transmit fluid is typically many orders of magnitude less than that of the fracture network (Cacas et al., 1990; Long et al., 1982; NAP, 2015). In this case, consideration of steady-state laminar flow of an incompressible fluid through a 1D parallel-plate smooth-walled fracture leads to the so-called cubic law for the fracture transmissivity $T\left[\mathrm{~m}^{2} \mathrm{~s}^{-1}\right]$ (Snow, 1969).

$$
T=\frac{\rho g}{12 \mu} b^{3}
$$

where $g$ is the acceleration of gravity $\left[\mathrm{m} \mathrm{s}^{-2}\right], \rho$ and $\mu$ are the density $\left[\mathrm{kg} \mathrm{m}^{-3}\right]$ and dynamic viscosity $\left[\mathrm{kg} \mathrm{m}^{-1} \mathrm{~s}^{-1}\right]$ of the fluid, respectively, and $b$ is the fracture aperture. To conduct our simulations, the DFN is first divided into fracture segments whose endpoints are formed by either (i) intersections between fractures and the domain boundaries; (ii) intersections between fractures themselves; or (iii) fracture extremities. The flow of water through each fracture segment is constant and determined solely by the product of the transmissivity and the negative hydraulic gradient, the latter of which is linear and given by the difference in hydraulic head between the segment endpoints divided by the length of the segment. This version of Darcy's law, combined with the principle of mass conservation at each fracture intersection, are used to construct a linear system whose unknowns are the values of the hydraulic head at the fracture intersections (de Dreuzy et al., 2001; Gisladottir et al., 2016; Long et al., 1982). Taking into account the boundary conditions imposed on the domain borders, we solve the linear system and use the resulting hydraulic head values to determine the flow through each of the fractures. 


\subsection{Electric current flow model}

Computing the flow of electric current through the DFN realizations is significantly more complicated than the fluid-flow problem because the contribution of the rock matrix cannot be ignored. Indeed, the smaller contrast in conductivity between the fractures and matrix in this case means that we must not only account for current flow through the matrix, but also between the fractures and matrix, in addition to the current flow through the fracture network. To this end, we use the discrete-dual-porosity approach of Roubinet \& Irving (2014) and refer the reader to this paper for details beyond the brief description given here. The equation that forms the basis for this approach, obtained by combining Ohm's law with the principle of conservation of electric charge at the point scale, is the following:

$$
-\nabla \cdot(\sigma \nabla V)=Q
$$

where $\sigma$ is the electrical conductivity $\left[\mathrm{S} \mathrm{m}^{-1}\right], \mathrm{V}$ is the electric potential [V], and $Q$ is a source (positive) or sink (negative) term $\left[\mathrm{C} \mathrm{m}^{-3} \mathrm{~s}^{-1}\right]$ that is used to account for charge movement between the fractures and matrix based on differences in their potential values.

To conduct the simulations, the matrix domain is divided into blocks at a chosen level of discretization and the DFN is again divided into fracture segments. This time, however, fracture-segment endpoints are defined by intersections between fractures and matrix-block boundaries in addition to the criteria given in Section 2.2. At the fracture-segment scale, equation (6) is used to derive an analytical expression for the 1D electric potential distribution along a segment, which depends on the potential values at the segment endpoints as well as on the potential value of the surrounding matrix block. In the simplified case of an insulating (zero conductivity) matrix, this expression reduces to a linear variation in potential between the endpoints, meaning that the electric current flow through the fracture can be obtained by multiplying the negative potential gradient with the electrical conductance $G[\mathrm{~S}]$ of the fracture, given by:

$$
G=\sigma_{f} b
$$


where $\sigma_{f}$ is the fracture electrical conductivity. We shall see later that this fundamental difference between electric current and fluid flow in an isolated fracture, namely the dependence of flow rate on aperture to the first power for electric current (equation (7)) versus aperture to the third power for fluid flow (equation (5)), contributes to significant differences between the REV size and tensor characteristics for the electrical and hydraulic conductivities.

Similar to the fluid-flow case, the analytical expression for the electric potential along a fracture segment is combined with the principle of charge conservation at the fracture-segment junctions in order to construct a linear system. This system, which has more unknowns than equations due to the addition of the unknown potential values of the matrix blocks, is completed through the consideration of equation (6) at the matrix-block scale using a finite-volume-type approach. Taking into account the boundary conditions imposed on the domain borders, we solve the full linear system for the potential values at the fracture-segment endpoints and in the matrix blocks, which allows us to compute the flow of electric current through the fractured region.

\subsection{Estimation of $2 \mathrm{D}$ conductivity tensor components}

To estimate the hydraulic and electrical conductivity tensor components corresponding to a particular DFN realization using the numerical models for fluid and electric current flow described above, we consider two orthogonal sets of Dirichlet boundary conditions having different fixed potential values on one set of opposing sides and a linear variation between these values on the other sides (Baghbanan \& Jing, 2007; Long et al., 1982; Min et al., 2004). These boundary conditions, illustrated in Figure 1c and 1d, have the effect of creating a linear potential gradient across the domain in the $x$ and $y$ directions, respectively. Assuming that the conductivity in the $2 \mathrm{D}$ domain can be represented by a second-order tensor, the resulting flux is given by

$$
\mathbf{q}=-\mathbf{C} \nabla \phi,
$$


where $\mathbf{q}$ is the flux vector (either $\left[\mathrm{m} \mathrm{s}^{-1}\right]$ or $\left[\mathrm{C} \mathrm{m}^{-2} \mathrm{~s}^{-1}\right]$ ), $\phi$ is the potential (either $[\mathrm{m}]$ or $[\mathrm{V}]$ ), and

$$
\mathbf{C}=\left[\begin{array}{ll}
C_{x x} & C_{x y} \\
C_{y x} & C_{y y}
\end{array}\right]
$$

is the conductivity tensor (either $\left[\mathrm{m} \mathrm{s}^{-1}\right]$ or $\left[\mathrm{S} \mathrm{m}^{-1}\right]$ ).

Taking the component of the flux through the domain in a particular direction having unit vector $\hat{\mathbf{u}}_{d}$ and dividing by the magnitude of the potential gradient, we define

$$
C_{d}=-\frac{\mathbf{q} \cdot \hat{\mathbf{u}}_{d}}{|\nabla \phi|}
$$

where $\cdot$ denotes the scalar product. Substitution of equation (8) into (10) yields

$$
C_{d}=-\frac{(-\mathbf{C} \nabla \phi) \cdot \hat{\mathbf{u}}_{d}}{|\nabla \phi|}=\hat{\mathbf{u}}_{d}^{T} \mathbf{C} \hat{\mathbf{u}}_{g}
$$

where $\hat{\mathbf{u}}_{g}$ is a unit vector in the direction of the potential gradient. It is clear from equation (11) that, using the boundary conditions illustrated in Figure 1c where the potential gradient is along the $x$ direction, measurement of $C_{d}$ in the $x$ and $y$ directions will yield estimates of conductivity tensor components $C_{x x}$ and $C_{y x}$, respectively. Conversely, considering the boundary conditions illustrated in Figure $1 \mathrm{~d}$ where $\hat{\mathbf{u}}_{g}$ is along the $y$ direction, measurement of $C_{d}$ in the $x$ and $y$ directions will provide estimates of components $C_{x y}$ and $C_{y y}$, respectively.

For each considered square domain size, we estimate $C_{x x}, C_{x y}, C_{y x}$, and $C_{y y}$ for multiple DFN realizations until stable estimates of the mean and variance of these tensor components across the realizations are obtained. Figure 2 shows an example of the progression of the mean and variance for the electrical conductivity as a function of the number of realizations considered, for a domain size of $12 \times 12 \mathrm{~m}$. The data presented in this figure correspond to one of the test cases investigated in Section 3. As could be expected, we see that the curves fluctuate quite significantly for low numbers of realizations, but then gradually approach fixed values as the number of samples increases. For this particular example, 100 generated DFNs appear to be sufficient to yield stable estimates of the mean and variance of the conductivity 
tensor components. This number will tend to increase for smaller domain sizes, and decrease for larger domains, as it depends on how well the domain size represents the REV.

[Figure 2 about here.]

We next examine the stable mean and variance estimates as a function of domain size in order to assess how these values vary with changing scale, with the aim of identifying the equivalent conductivity tensor for the medium and ultimately the size of the REV. Figure 3 shows an example of the values obtained for the electrical conductivity, again for one of the test cases investigated in Section 3, for domains having side lengths of $L=4,8,12$, and $16 \mathrm{~m}$. Note that the results shown in this figure are typical of our findings in each test case. Quite importantly, we see that with the exception of the smallest domain size $(L=4 \mathrm{~m})$, the mean values for the tensor components are consistent, suggesting that they will not change as the domain gets larger and are thus representative of the medium's large-scale effective behaviour. That is, although a single DFN realization may yield values of components $C_{x x}, C_{x y}, C_{y x}$, and $C_{y y}$ that are far from the equivalent tensor values for the medium, and indeed which may not even correspond to an anisotropic conductivity tensor in the sense that $C_{x y} \neq C_{y x}$, the mean across multiple realizations will provide reasonable estimates of these components (Kanit et al., 2003). As a result, we use the mean tensor values for the largest domain size considered in our analysis $(L=16 \mathrm{~m})$ to determine the large-scale tensorial properties of the fracture network corresponding to a chosen statistical distribution of fracture positions, orientations, lengths, and apertures. The eigenvalues and eigenvectors of the equivalent tensor matrix are used to determine the maximum and minimum conductivity values and principal directions, respectively (Bear, 2013). With regard to the variability of the estimated tensor components, we see in Figure 3 that the standard deviations decrease as the domain size increases, which is expected because larger domains will better represent the overall medium properties. As discussed in the next section, the trend in conductivity variability with domain size allows us to establish a scaling relationship that is used to estimate the size of the REV.

[Figure 3 about here.] 


\subsection{Determination of the REV size}

Cailletaud et al. (1994) and Kanit et al. (2003) proposed a combined statistical and numerical approach to the REV estimation problem, whereby a scaling relationship for the variance of the parameter of interest is established based on numerical simulations in order to define the size of the REV in terms of a prescribed level of statistical error. Originally used by the authors to investigate the bulk thermal and elastic properties of random composites, the approach has since been applied to a variety of other problems, including examination of the elastic and electrical behaviour of multi-scale high-contrast materials (Willot \& Jeulin, 2011), quantification of the matrix clay content of rocks at the mesoscale (Keller, 2015), and the upscaling of seismic P- and S-wave moduli in fractured media (Caspari et al., 2016). A key tenet of the approach holds that the notion of a single REV size for a heterogeneous material should be abandoned. Instead, it is argued that the size of the REV must be considered within a statistical framework as its value will depend upon the level of error in the largescale equivalent properties deemed acceptable, the physical property being considered and, in the case where the goal is estimation of the minimum domain size required to determine the macroscopic properties of the medium, the number of samples or realizations at that domain size that are available.

In our work, we wish to determine the minimum volume of fractured rock for which the electrical or hydraulic conductivity exhibited by that volume is representative of the equivalent tensor properties of the fractured domain, to within some level of error $\epsilon$. To simplify our analysis, we do not examine the variability of each conductivity tensor component individually, but rather that of the first invariant of the conductivity tensor given by $I_{C}=C_{x x}+C_{y y}$ (Li et al., 2009; Li \& Zhang, 2010). The strong advantage of working with the invariant is that it is independent of the chosen coordinate system. Therefore the results obtained will not depend upon the orientation of the fracture network with respect to the applied boundary conditions. Assuming that $I_{C}$ for a particular domain area $S$ is a Gaussian distributed random variable with mean $E\left\{I_{C}(S)\right\}$ and variance $\operatorname{Var}\left\{I_{C}(S)\right\}$, a particular DFN realization at that domain size will have, with a $95 \%$ degree of confidence, an $I_{C}$ value lying within a distance $\epsilon$ of the mean when $\epsilon=2 \sqrt{\operatorname{Var}\left\{I_{C}(S)\right\}}$. In terms of the relative error $\epsilon_{r}$, this can be expressed 


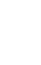

Var $\left\{I_{C}(S)\right\}$ changes as a function of $S$, which is obtained by fitting an assumed form of scaling relationship to the results of our numerical simulations on DFNs of various sizes.

Based on previous work (Cailletaud et al., 1994; Kanit et al., 2003; Lantuejoul, 1991), we postulate that the following power-law scaling relationship applies to the first invariant of the hydraulic and electrical conductivity tensors:

$$
\operatorname{Var}\left\{I_{C}(S)\right\}=\kappa S^{-\alpha}
$$

where $\kappa$ and $\alpha$ are fitting parameters that depend on the nature of the fracture network and physical property being studied. For properties such as the volumetric average or volume fraction, which represent an additive combination of the small-scale medium heterogeneities, classical geostatistical theory predicts that $\alpha=1$ and that $\kappa$ will be equal to the product of the medium's integral range and the point-scale property variance (Chiles \& Delfiner, 1999; Lantuejoul, 1991; Matheron, 1971). Properties like the electrical and hydraulic conductivity, however, are not additive meaning that, in general, $\alpha \neq 1$. Although the form of equation (13) cannot be proven for the electrical and hydraulic conductivities in fractured rock, a number of empirical studies have shown the suitability of this relationship for similar non-additive physical quantities (Cailletaud et al., 1994; Kanit et al., 2003; Lantuejoul, 1991; Willot \& Jeulin, 2011). Furthermore, all of the test cases examined in Section 3 suggest that use of the power-law relationship is appropriate.

Substituting equation (13) into (12) and setting $S=L^{2}$, we estimate the size of the REV in terms of the square side length $L$ as follows:

$$
L=\left(\frac{2 \sqrt{\kappa}}{\epsilon_{r} E\left\{I_{C}(S)\right\}}\right)^{1 / \alpha}
$$


To determine parameters $\kappa$ and $\alpha$, we (i) estimate the variance of $I_{C}$ from the results of our numerical flow simulations on multiple DFN realizations for each studied domain size; (ii) make a log-log plot of $\operatorname{Var}\left\{I_{C}(S)\right\}$ versus $S$; and (iii) determine the slope and intercept of the corresponding least-squares best-fitting line through the points. As an example, Figure 4 shows a log-log plot of the variance of $I_{C}$ versus domain area $L^{2}$ for the electrical conductivity. Again, the results shown in this figure are typical of the different test cases considered in our study. We see that the points tend to fall along a straight line, whose slope and intercept allow us to estimate $\alpha$ and $\kappa$, respectively.

\section{[Figure 4 about here.]}

\section{Results}

\subsection{Test cases and analysis}

We now apply the analysis methodology presented in Section 2 to a total of 16 different test cases, where our goal is to examine how changes in the parameters governing the fracture distribution affect the REV size and equivalent tensor characteristics for the electrical and hydraulic conductivities. Table 1 summarizes each test case in terms of the angle, aperture, and length distributions considered for the two fracture sets. In Figure 5, we show example DFNs corresponding to each case for a $16 \times 16 \mathrm{~m}$ domain size, where the colour of the lines is used to quantify the fracture aperture. Finally, Figure $6 \mathrm{a}$ and $6 \mathrm{~b}$ show histograms of the two truncated log-normal probability distributions for the aperture considered in Cases 12, 13, and 14, whereas Figure 6c and 6d show histograms of the fracture length distributions for power-law exponent values of $a=2.0$ and $a=2.5$, respectively.

\section{[Table 1 about here.]}

[Figure 5 about here.]

[Figure 6 about here.]

For the electrical conductivity, two scenarios are considered in our analysis. In the first scenario, which we believe to be most representative of real-world conditions, we assume a 
rock matrix conductivity of $\sigma_{m}=10^{-4} \mathrm{~S} \mathrm{~m}^{-1}$ and a fracture or groundwater conductivity of $\sigma_{f}=10^{-1} \mathrm{~S} \mathrm{~m}^{-1}$ (Schön, 2015). Here, the matrix plays an important and normal role in the conduction of electric current through the rock as the ratio between the conductivities is $\sigma_{m} / \sigma_{f}=10^{-3}$ (Roubinet \& Irving, 2014). In the second scenario, the matrix conductivity is decreased to a value of $\sigma_{m}=10^{-7} \mathrm{~S} \mathrm{~m}^{-1}$ such that the contribution of the matrix to electrical conduction through the rock is negligible. The goal with this scenario, where $\sigma_{m} / \sigma_{f}=10^{-6}$, is to have a test situation that allows us to assess the impact of the single power versus cubic dependence on aperture of the fracture conductance and transmissivity, respectively, as well as examine the role of the matrix on the REV size and equivalent tensor characteristics.

In our analysis, square domain sizes of $L=4,8,12$, and $16 \mathrm{~m}$ were considered for each test case to calibrate the scaling relationship for the tensor invariant in equation (14). A relative error of $\epsilon_{r}=20 \%$ was considered in our estimation of the REV size (Li et al., 2009; Li \& Zhang, 2010). The mean tensor components for the largest $(16 \times 16 \mathrm{~m})$ domain size were used to estimate the equivalent conductivity tensor. The number of realizations needed to obtain stable mean and variance estimates of each tensor component was chosen as the point at which the cumulative values did not vary more than $5 \%$ over the last 20 samples (Figure 2). For the fluid and electric current flow modeling, hydraulic head and electric potential differences of $1 \mathrm{~m}$ and $1 \mathrm{~V}$ were applied across each considered DFN (Figure 1c and 1d). For the electric current flow modeling, the rock matrix was discretized into square blocks of side length $2 \mathrm{~m}$.

Table 2 shows the results obtained for each test case for the electrical conductivity $(\sigma)$ assuming $\sigma_{m} / \sigma_{f}=10^{-3}$, whereas Table 3 shows the results obtained assuming $\sigma_{m} / \sigma_{f}=10^{-6}$. The corresponding results for the hydraulic conductivity $(K)$ are given in Table 4 . In the tables we provide (i) the estimated REV size; (ii) the maximum and minimum principal values of the conductivity tensor; (iii) the corresponding conductivity anisotropy ratio; and (iv) the direction of maximum conductivity. Note that the direction of maximum conductivity is only given if the anisotropy is greater than $5 \%$. Otherwise, the system is considered to be effectively isotropic with no preferred orientation. Below we discuss how all of these quantities compare between the electrical and hydraulic conductivities, as well as vary as we change details of the statistical distributions for the fracture orientations, apertures, and lengths. 
[Table 2 about here.]

[Table 3 about here.]

[Table 4 about here.]

\subsection{Effect of changing angle between the fracture sets}

We first consider Cases 1, 2, and 3 from Table 1, whereby the angle between the two fracture sets is changed by varying the mean orientation angle of Fracture Set 2. Figure 7 shows the impact of this change on the REV size (Figure 7a), the anisotropy ratio of the equivalent conductivity tensor (Figure 7b), the direction of maximum conductivity (Figure 7c), and the maximum conductivity value (Figure $7 \mathrm{~d}$ ). Note that the maximum conductivities plotted in Figure $7 \mathrm{~d}$ were normalized by the values obtained for Case 1 in order to better compare the relative changes between the hydraulic and electrical conductivities.

[Figure 7 about here.]

We see in Figure 7a that, in accordance with previous work (Wang \& Kulatilake, 2008), the size of the REV for the hydraulic conductivity decreases as the angle between the two fracture sets increases from 30 to 90 degrees. The curve for the electrical conductivity with $\sigma_{m} / \sigma_{f}=10^{-6}$ is observed to exhibit exactly the same trend. This occurs because, as the range of fracture orientations in the medium increases, smaller domain sizes become able to support flow in all directions through the fracture network, thereby allowing those domain sizes to better represent the conductivity as an equivalent tensor. Indeed, in a fracture network where the angle between the two fracture sets is small, connectivity in all directions through the network will only be established for larger domain sizes because smaller domains will not allow sides of the network that are largely parallel to the fractures to be connected via the fractures. This implies a larger REV size. For the electrical conductivity scenario with $\sigma_{m} / \sigma_{f}=10^{-3}$, on the other hand, Figure 7a shows that the REV size is noticeably smaller and remains approximately constant as the angle between the fracture sets is increased. Quite importantly, the higher electrical conductivity of the matrix in this scenario has a strong homogenizing effect, meaning that the fractures are less critical for current flow through the 
domain and the medium can thus be represented by an equivalent tensor at a $30-50 \%$ smaller scale. This strong reduction in the REV size for the $\sigma_{m} / \sigma_{f}=10^{-3}$ scenario, which we again believe to be more representative of realistic conditions than the $\sigma_{m} / \sigma_{f}=10^{-6}$ scenario in the sense that conduction through the rock matrix cannot be ignored, occurs in all of the 16 test cases considered in our study (Tables 2 and 3). It has strong practical implications because it means that (i) the electrical conductivity can always be modeled as an equivalent tensor at a notably smaller scale than the hydraulic conductivity; and (ii) this tensor, for reasons of scale alone, is not likely to easily translate to the hydraulic conductivity.

Regarding the anisotropy of the equivalent conductivity tensor, we see in Figure $7 \mathrm{~b}$ that, as expected, the degree of anisotropy tends to decrease as the angle between the two fracture sets increases from 30 to 90 degrees, with the case of two orthogonal fracture sets being effectively isotropic (Wang \& Kulatilake, 2008). This decrease is seen to be greatest for $K$ and for the $\sigma_{m} / \sigma_{f}=10^{-6}$ scenario because the connectivity in these situations is controlled completely by the fractures. Because the apertures of Fracture Sets 1 and 2 are the same and there is an approximately equal number of fractures belonging to each set in the domain, the direction of maximum conductivity in all cases is seen to take the average of the mean orientations of these fracture sets (Figure 7c). The maximum conductivity in Figure $7 \mathrm{~d}$ is observed to decrease slightly as the angles of Fracture Sets 1 and 2 diverge, with the changes being less pronounced when $\sigma_{m} / \sigma_{f}=10^{-3}$.

\subsection{Effect of changing fracture angle variability}

We next examine Cases 2 and 4, where the variability of the orientation angle of both fracture sets is increased by changing the standard deviation from 5 to 10 degrees, respectively. The average angle between the fracture sets in both cases is 60 degrees (Table 1). Figure 8 shows the corresponding results where we see that, despite that fact that the orientation angle variability is doubled between Cases 2 and 4, there are minor changes in the REV size, equivalent tensor anisotropy ratio, direction of maximum conductivity, and maximum conductivity value for both the hydraulic and electrical conductivities. Although this result is surprising given the findings of Long et al. (1982) and Wang \& Kulatilake (2008), who saw 
a reduction in the REV size and degree of anisotropy for the permeability with an increase in fracture orientation variability, it likely occurs because the range of angles covered by the fracture network in Case 2 is already quite extensive, and thus not significantly changed when the spread of the orientation angle of each fracture set is increased. Indeed, a comparison of the example DFNs for Cases 2 and 4 suggests that the differences in fracture-network connectivity are rather minimal (Figure 5). Again, we observe in Figure 8a that the REV size is $42-46 \%$ smaller for the $\sigma_{m} / \sigma_{f}=10^{-3}$ scenario. The degree of anisotropy is also less for this scenario (Figure 8b) because of the homogenizing effect of the matrix conductivity.

\section{[Figure 8 about here.]}

\subsection{Effect of changing fracture aperture}

In the next series of tests, we examine how changing the aperture of the fracture sets impacts the REV size and properties of the equivalent tensors for $K$ and $\sigma$. We first consider Cases 2 and 11, between which the aperture of Fracture Sets 1 and 2 is increased from $1 \mathrm{~mm}$ to $1.5 \mathrm{~mm}$ (Table 1). Figure 9 shows the corresponding results. We see in Figure 9a that, for the hydraulic conductivity and for the electrical conductivity when $\sigma_{m} / \sigma_{f}=10^{-6}$, a uniform increase in the fracture aperture has no impact on the REV size. This is because the domain properties are controlled completely by the fractures in these two situations, and uniformly increasing the flow through all of the fractures by changing their aperture should not affect the scale at which the fracture network can be effectively described by an anisotropic tensor. It will, however, increase the overall magnitude of the tensor components, which is clearly shown in Figure 9d with the largest relative change exhibited by $K$ because of the cubic dependence of fracture hydraulic transmissivity on aperture (equation (5)). For the electrical conductivity when $\sigma_{m} / \sigma_{f}=10^{-3}$, on the other hand, increasing the fracture aperture is seen to cause in a slight increase in the REV size. This occurs because the fractures account for a greater fraction of the total current flow through the rock when their aperture is larger, and thus the previously described homogenizing effect of the matrix conductivity, which again tends to decrease the REV size, is reduced. The lesser importance of matrix current flow with larger aperture also translates to a slight increase in the tensor anisotropy ratio for the 
$\sigma_{m} / \sigma_{f}=10^{-3}$ scenario (Figure $9 \mathrm{~b}$ ).

[Figure 9 about here.]

Next we consider Cases 2, 8, 9, and 10, where we investigate the effects of increasing the aperture of Fracture Set 2 while keeping the aperture of Fracture Set 1 fixed at $1 \mathrm{~mm}$. The average angle between the fracture sets is 60 degrees (Table 1). Figure 10 shows the results obtained for the REV size and equivalent conductivity tensor as a function of the aperture of Fracture Set 2. We see in Figure 10a that, as the aperture increases, the size of the REV also increases because the second fracture set gradually begins to dominate the flow response, making the medium behave more like one having only a single set of fractures. The increase in REV size is greatest for $K$ because of the cubic dependence of hydraulic transmissivity on aperture, whereas for $\sigma_{m} / \sigma_{f}=10^{-6}$ only a small change is observed. For the electrical conductivity scenario where $\sigma_{m} / \sigma_{f}=10^{-3}$, the increase in REV size is slightly greater because the fractures carry a larger fraction of the total current flow compared to the matrix as the aperture of Fracture Set 2 is increased.

[Figure 10 about here.]

With regard to the equivalent conductivity tensor characteristics, the change in aperture of Fracture Set 2 is seen to have significant effects on the anisotropy ratio (Figure 10b), the direction of maximum conductivity (Figure 10c), and the maximum conductivity value (Figure 10d), with the changes for $K$ being greater than those for $\sigma$, again because of the much stronger dependence of fluid flow upon aperture through the cubic law. In Figure 10b, we see that the anisotropy ratio for $K$ first decreases as the aperture of Fracture Set 2 is increased from $1 \mathrm{~mm}$ to $1.1 \mathrm{~mm}$, but then increases sharply as the aperture approaches $2 \mathrm{~mm}$. This can be explained by the fact that, when the apertures of Fracture Sets 1 and 2 are equal (Case 2) there is already substantial anisotropy along a 30-degree orientation exhibited by the equivalent $K$ tensor. As the aperture of Fracture Set 2, which has a mean orientation of 60 degrees, is increased (Case 8), the initial tendency is to reduce the existing anisotropy by stretching the tensor ellipse away from the direction of maximum conductivity. When the aperture is increased further, however, Fracture Set 2 eventually dominates the flow response 
(Case 10), causing strong anisotropy along a different, 60-degree orientation. With regard to $\sigma$, the same general trend with increasing aperture is observed except that, because of the weaker dependence of electric current flow upon aperture compared to fluid flow, a $2 \mathrm{~mm}$ aperture for Fracture Set 2 (Case 10) is not yet large enough for this fracture set to dominate the flow response and cause anisotropy along the 60-degree orientation. As a result, we see only the system becoming more isotropic as the aperture of Fracture Set 2 is increased.

In Figure 10c, we observe one of the most important results of our analysis, which is that the maximum principal direction of the equivalent tensor can be significantly different between the hydraulic and electrical conductivities when the aperture distribution between the fracture sets is not the same. When both fracture sets share the same 1-mm constant aperture, for example, the directions of maximum conductivity for $K$ and $\sigma$ are seen to be identical and equal to 30 degrees, the average value between the mean orientations of the two fracture sets. As the aperture of Fracture Set 2 is increased, however, the principal orientations between $K$ and $\sigma$ diverge because fluid flow through the domain is affected much more than electric current flow, meaning that the maximum principal direction of the $K$ tensor moves more quickly towards the 60-degree orientation. This finding, which is admittedly rather intuitive, has significant implications for studies where researchers have attempted to infer principal groundwater flow directions from the results of azimuthal resistivity surveys in fractured rock (Ritzi \& Andolsek, 1992; Skinner \& Heinson, 2004; Skjernaa \& Jørgensen, 1994; Steinich \& Marin, 1996; Yeboah-Forson \& Whitman, 2014). Specifically, our results indicate that only in very specific circumstances can the principal directions of the electrical conductivity tensor be expected to provide meaningful information regarding those of the hydraulic conductivity tensor. Further, the ratio and trends observed in the maximum and minimum principal conductivity values will, in general, vary significantly between $K$ and $\sigma$ (Figure 10b and 10d).

Finally, we consider Cases 3,5,6, and 7, where we again increase only the aperture of Fracture Set 2, but this time considering an average angle between the fracture sets of 90 degrees. Figure 11 shows the corresponding results for the REV size and equivalent conductivity tensor characteristics. As we observed in Figure 10, the REV size for $K$ is seen to increase with an increase in the aperture of Fracture Set 2 because this fracture set quickly 
begins to dominate the flow response as a result of the cubic law. The changes in REV size for the $\sigma_{m} / \sigma_{f}=10^{-3}$ and $\sigma_{m} / \sigma_{f}=10^{-6}$ scenarios, on the other hand, are again rather negligible (Figure 11a). As there is no anisotropy when the apertures of the two fracture sets are equal (Case 3), the anisotropy ratio is seen to consistently increase as the aperture of Fracture Set 2 increases (Figure 11b), with the direction of maximum conductivity in all cases being approximately equal to 90 degrees, which is the mean orientation of the second fracture set (Figure 11c). In other words, making the aperture of Fracture Set 2 larger in this case immediately results in anisotropy along the 90-degree orientation, with the greatest increase in anisotropy being exhibited by $K$. Similarly, the maximum conductivity value is seen to increase most for $K$ with an increase in the aperture of the second fracture set (Figure 11d), which again results from the greater sensitivity of fluid flow to fracture aperture as compared to electric current flow.

[Figure 11 about here.]

\subsection{Effect of changing fracture aperture variability}

We now investigate how changing the variability of the aperture distribution of both fracture sets affects the REV size and equivalent tensor characteristics for the electrical and hydraulic conductivities. To this end, we examine Cases 2,12 , and 13 , which involve a constant aperture and two truncated log-normal aperture distributions having different spreads (Table 1). The histograms for the log-normal distributions considered in Cases 12 and 13 are shown in Figure $6 \mathrm{a}$ and $6 \mathrm{~b}$, respectively, where we see that the choice of parameters for the mean and variance of the natural logarithm of the aperture are such that the peak value remains constant and equal to $1 \mathrm{~mm}$, but the dispersion around this value changes. Figure 12 shows the corresponding results. In Figure 12a we observe that, as the spread of the aperture distribution of both fracture sets increases, the REV size for $K$ increases, whereas the REV sizes for the $\sigma_{m} / \sigma_{f}=10^{-3}$ and $\sigma_{m} / \sigma_{f}=10^{-6}$ scenarios exhibit minimal changes. Again, because of the strong dependence of hydraulic transmissivity on fracture aperture compared to the electrical conductance, the fluid flow behaviour of the network will be greatly influenced by randomly distributed, large-aperture fractures, which become more prevalent when 
the fracture aperture spread is increased (Figure 5). To account for this increased variability, the domain size required to represent the flow response using an equivalent $K$ tensor must increase (Baghbanan \& Jing, 2007; Long et al., 1982). Note, however, that no meaningful changes in the anisotropy ratio and principal directions of the $K$ and $\sigma$ tensors are observed as the spread of the aperture distribution increases (Figure $12 \mathrm{~b}$ and $12 \mathrm{c}$ ). This is because the same aperture distribution was considered for the two fracture sets in Cases 2, 12, and 13, and there is no reason to expect that changes in this distribution would lead to changes in the medium anisotropic characteristics. Conversely, we see in Figure $12 \mathrm{~d}$ that an increased variability in fracture aperture results in an increase in the overall conductivity magnitude, as the presence of a greater number of large-aperture fractures will increase the amount of fluid and electric current flow through the domain. This is most significant for $K$ because of the cubic law.

[Figure 12 about here.]

\subsection{Effect of correlation between aperture and length}

Our second-last test involves examination of the impact of correlation between fracture aperture and length on the REV size and equivalent tensor properties. As mentioned previously, there is significant empirical and theoretical evidence to support fracture apertures being positively correlated with their length. This prompted an investigation into the effects of correlation between these two variables on the REV size and tensor characteristics for the permeability (Baghbanan \& Jing, 2007), but never before for the electrical conductivity. In this regard, we now consider Cases 12 and 14, which involve the same truncated log-normal distribution for the fracture aperture in the absence and presence of correlation between aperture and length, respectively. Figure 13 shows the results obtained, where correlation is indicated on the horizontal axis using a binary variable $(0=$ uncorrelated; $1=$ correlated $)$. We see in the figure that, as expected, correlation between aperture and length has no impact on the anisotropic characteristics of the equivalent $K$ and $\sigma$ tensors (Figure 13b and 13c). However, in accordance with Baghbanan \& Jing (2007), it does lead to an increase in the REV size (Figure 13a) as well as the overall conductivity magnitudes (Figure 13d). The latter find- 
ings are explained by the fact that correlation between aperture and length means that longer fractures in the domain will be associated with larger apertures (Figure 5), which greatly increases the probability that the fluid and electric current flow behaviour will be dominated by a small number of long fractures, as opposed to being more equally influenced by all of the fractures in the network. As a result, the size of the REV must increase to accommodate the increased variability in the flow response, with the change in REV size being greatest for $K$ and for $\sigma_{m} / \sigma_{f}=10^{-6}$ where flow through the matrix is negligible. The overall conductivity magnitudes must also increase because flow through the domain will be facilitated by the long, large-aperture fractures, especially for $K$.

[Figure 13 about here.]

\subsection{Effect of changing fracture length power-law exponent}

Finally, we examine the impact of the fracture length power-law exponent $a$ from equation (1). To this end, we consider Cases 2, 15, and 16, where $a$ is increased from 2.0 to 2.5 while keeping the other fracture-network parameters constant (Table 1). Figure 6c and 6d show histograms of the fracture length distribution for values of $a=2.0$ and $a=2.5$, respectively, where we see that increasing the power-law exponent results in a greater proportion of shorter fractures throughout the domain (see also Figure 5). The impact of this change on the REV size and equivalent $K$ and $\sigma$ tensor characteristics is shown in Figure 14. We observe in Figure 14a that, as the value of $a$ increases and connectivity across the domain becomes dependent upon a smaller number of randomly distributed long fractures, the REV sizes for $K$ and for the $\sigma_{m} / \sigma_{f}=10^{-6}$ scenario increase by a factor of approximately 5 . As flow occurs purely through the fracture network, a larger domain size is required to accommodate the greater variability in flow behaviour and represent the network as an equivalent tensor quantity. Conversely, for the electrical conductivity when $\sigma_{m} / \sigma_{f}=10^{-3}$, there is only a slight increase in the REV size with increasing $a$ value (Table 2) because the electric current flow through the matrix permits connections across the domain independently of the fracture network, thereby reducing the importance of the latter. With regard to the tensor anisotropy ratio, Figure 14b shows a gradual decrease with increasing power-law exponent, which likely results because the smaller 
number of long fractures tends to reduce the directionality of the flow response. As expected, the direction of maximum conductivity is not affected by the change in power-law exponent (Figure 6c) and is equal, as before, to the average value of the mean orientations of the two fracture sets. Finally, in Figure 14d we see that increasing the fracture length power-law exponent has the effect of reducing the maximum principal conductivity value for both $K$ and $\sigma$, as connectivity across the domain is notably reduced. Because of the contribution of the matrix, this reduction is less pronounced for the $\sigma_{m} / \sigma_{f}=10^{-3}$ scenario.

[Figure 14 about here.]

\section{Discussion and conclusions}

We have presented in this paper a systematic analysis of the effects of changes in the statistical parameters governing fracture networks on the REV size and equivalent tensor characteristics for the electrical and hydraulic conductivities. Thanks to the recently developed DDP modeling approach of Roubinet \& Irving (2014), electric current flow through arbitrarily complex fractured domains can be simulated efficiently and accurately, properly taking into account important contributions to current flow through the matrix as well as between the fractures and matrix. Groundwater flow, on the other hand, was simulated in our work using a standard DFN methodology based on the usual assumption that matrix fluid flow is negligible due to the many-orders-of-magnitude difference between the hydraulic properties of the matrix and those of the fractures. One strong advantage of the combined statistical and numerical analysis approach considered in this paper, which builds on the seminal work of Cailletaud et al. (1994) and Kanit et al. (2003), is that numerical flow simulations need not be performed at the REV scale in order to estimate the REV size and equivalent tensor properties. That is, we can conduct the entire REV analysis using smaller, less computationally burdensome, domain sizes whose statistical characteristics can then be linked to those of the REV. The approach also has the advantage of explicitly treating the REV size as a stochastic quantity, whose estimated value depends upon the error in large-scale equivalent properties that one is willing to accept. Although these advantages do come at the cost of needing to define in 
advance a scaling relationship for the conductivity variability, all of the results obtained in this paper suggest that our assumption of power-law scaling is appropriate.

Two key differences between groundwater and electric current flow in fractured rock were seen to lead to significant differences in the REV size and tensor characteristics between the electrical and hydraulic conductivities. First, matrix flow must be considered in the electrical case, which tends to have a homogenizing effect on the flow response in the sense that, because the matrix is responsible for a significant fraction of the total current passing through the rock, the effect of the fractures will be less pronounced. Secondly, whereas the hydraulic transmissivity of a fracture varies with the cube of its aperture, the analogous electrical conductance varies only linearly with the aperture. As a result of these two differences we observed that: (i) the REV size and degree of anisotropy are consistently less for the electrical conductivity than for the hydraulic conductivity for realistic matrix-to-fracture conductivity ratios (e.g., the considered $\sigma_{m} / \sigma_{f}=10^{-3}$ scenario); (ii) changes in the angle, aperture, and length distributions of the fracture network have a stronger effect on $K$ than on $\sigma$, in particular with regard to the aperture; and (iii) in the presence of more than one fracture set, the principal directions of the equivalent electrical conductivity tensor do not generally correspond with those of the equivalent hydraulic conductivity tensor, and in fact can vary by a significant amount. Consequently, attempting to make conclusions about the hydrogeological properties of fractured domains based on geoelectrical measurements, as has been attempted in many previous studies, must be done with great caution. It cannot, for example, be generally assumed that the degree and direction of electrical conductivity anisotropy, estimated from an azimuthal resistivity survey, will provide useful information on hydraulic anisotropy. Further, changes observed in $\sigma$ across a domain cannot be interpreted in terms of changes in $K$, except in a highly qualitative manner. Finally, variations in fracture aperture that have a strong effect on fluid flow may have minimal influence on geoelectrical measurements, meaning that ER data will be of limited use to assess many important hydraulic characteristics. Note that, although our results were obtained for the DC resistivity, we expect them to be equally applicable to EM-based investigations, where directional properties of the conductivity at low frequencies have been interpreted hydrogeologically (Steelman et al., 2015). 
It is important to emphasize that, for all of the analyses carried out in this paper, we considered boundary conditions corresponding to a linear potential gradient across the fractured domain, which are fully consistent with previous hydrogeological research aimed at assessing the intrinsic equivalent properties of the fracture network along with the REV size (Baghbanan \& Jing, 2007; Long et al., 1982; Min et al., 2004; Wang \& Kulatilake, 2008). Although such linear potential gradients are likely to exist away from point sources such as pumping or injection wells and current electrodes, boundary conditions in the vicinity of these sources will differ and therefore also the flow of water or electric current through the fracture network. As a result, an important topic of future research is the investigation of how the key differences between the hydraulic and electrical conductivities highlighted in this work are manifested in real-world field experiments to measure these properties, which typically involve pumping/injection experiments and the use of point electrodes, respectively. To this end, we are currently developing numerical modeling codes for the accurate simulation of azimuthal resistivity measurements in 3D fractured-rock environments.

We also note that the numerical values for the REV size determined in this paper are not nearly as important as the trends in REV size observed as a function of changes in fracture-network properties, as well as how results compare between the hydraulic and electrical conductivities. Indeed, the estimated REV size depends on many factors, most notable of which are the fracture density and prescribed level of error in equivalent properties considered to be acceptable. Further, we have not addressed in this paper the question of the existence of the REV, which may not occur for some fracture networks or may happen at a variety of different scales (Long et al., 1982). It should also be pointed out that, as much as we have considered the effects of changing a variety of fracture properties (i.e., orientation, aperture, length) as well as the matrix-to-fracture electrical conductivity ratio in this paper, it is the overall difference between the effective fracture network conductance, which depends on all of these factors, and the matrix conductance that will tend to control the homogenizing effects observed. Finally, as in other related DFN studies for the permeability, we have not examined in this paper the effects of aperture variability within a fracture and the impact of fracture filling or alteration. Initial investigations into these issues could be performed 
792

with the considered DDP and DFN numerical modeling approaches by dividing individual fractures into sub-fractures having different properties. This is a topic of future work.

\section{Acknowledgements}

This work was funded by a grant to J. Irving from the Swiss National Science Foundation (Number 200021_143758). 


\section{References}

Al Hagrey, S. A., 1994. Electric study of fracture anisotropy at Falkenberg, Germany, Geophysics, 59(6), 881-888.

Baghbanan, A. \& Jing, L., 2007. Hydraulic properties of fractured rock masses with correlated fracture length and aperture, International Journal of Rock Mechanics and Mining Sciences, 44(5), 704-719.

Bear, J., 2013. Dynamics of fluids in porous media, Courier Corporation.

Berkowitz, B., 2002. Characterizing flow and transport in fractured geological media: A review, Advances in Water Resources, 25(8-12), 861-884.

Berryman, J. G. \& Hoversten, G. M., 2013. Modelling electrical conductivity for earth media with macroscopic fluid-filled fractures, Geophysical Prospecting, 61(2), 471-493.

Beskardes, G. D. \& Weiss, C. J., 2018. Modelling DC responses of 3D complex fracture networks, Geophysical Journal International.

Boadu, F., Gyamfi, J., \& Owusu, E., 2005. Determining subsurface fracture characteristics from azimuthal resistivity surveys: A case study at Nsawam, Ghana, Geophysics, 70(5), B35-B42.

Boadu, F. K., Owusu-Nimo, F., \& Menyeh, A., 2008. Nitrate contamination in groundwater at farmlands in Nsawam, Ghana: the role of fractures from azimuthal resistivity surveys, Journal of Environmental \& Engineering Geophysics, 13(1), 27-37.

Bonnet, E., Bour, O., Odling, N. E., Davy, P., Main, I., Cowie, P., \& Berkowitz, B., 2001. Scaling of fracture systems in geological media, Reviews of Geophysics, 39(3), 347-383.

Bour, O. \& Davy, P., 1997. Connectivity of random fault networks following a power law fault length distribution, Water Resources Research, 33(7), 1567-1583.

Brown, S. R., 1989. Transport of fluid and electric current through a single fracture, Journal of Geophysical Research: Solid Earth, 94(B7), 9429-9438.

Busby, J., 2000. The effectiveness of azimuthal apparent-resistivity measurements as a method for determining fracture strike orientations, Geophysical Prospecting, 48(4), 677-695.

Caballero Sanz, V., Roubinet, D., Demirel, S., \& Irving, J., 2017. 2.5-D discrete-dual-porosity model for simulating geoelectrical experiments in fractured rock, Geophysical Journal International, 209(2), 1099-1110.

Cacas, M., Ledoux, E., de Marsily, G., Tillie, B., Barbreau, A., Durand, E., Feuga, B., \& Peaudecerf, P., 1990. Modeling fracture flow with a stochastic discrete fracture network: Calibration and validation: 1. The flow model, Water Resources Research, 26(3), doi:10.1029/WR026i003p00479.

Cailletaud, G., Jeulin, D., \& Rolland, P., 1994. Size effect on elastic properties of random composites, Engineering Computations, 11(2), 99-110. 
Carneiro, J. F., 2009. Numerical simulations on the influence of matrix diffusion to carbon sequestration in double porosity fissured aquifers, International Journal of Greenhouse Gas Control, 3(4), 431-443.

Caspari, E., Milani, M., Rubino, J., Müller, T. M., Quintal, B., \& Holliger, K., 2016. Numerical upscaling of frequency-dependent P- and S-wave moduli in fractured porous media, Geophysical Prospecting, 64(4), 1166-1179.

Chiles, J. P. \& Delfiner, P., 1999. Geostatistics: Modeling Spatial Uncertainty, John Wiley \& Sons, New York.

Cvetkovic, V., 2017. Statistical formulation of generalized tracer retention in fractured rock, Water Resources Research, 53(11), 8736-8759.

Davy, P., Le Goc, R., Darcel, C., Bour, O., De Dreuzy, J.-R., \& Munier, R., 2010. A likely universal model of fracture scaling and its consequence for crustal hydromechanics, Journal of Geophysical Research: Solid Earth, 115(B10).

de Dreuzy, J.-R., Davy, P., \& Bour, O., 2001. Hydraulic properties of two-dimensional random fracture networks following a power law length distribution: 1. Effective connectivity, Water Resources Research, 37(8), 2065-2078.

DesRoches, A. J., Butler, K. E., \& MacQuarrie, K. T., 2017. Surface self-potential patterns related to transmissive fracture trends during a water injection test, Geophysical Journal International, 212(3), 2047-2060.

Devroye, L., 1986. Non-Uniform Random Variate Generation, Springer-Verlag.

Dey, A. \& Morrison, H., 1979. Resistivity modeling for arbitrarily shaped two-dimensional structures, Geophysical Prospecting, 27(1), 106-136.

Donadille, J.-M. \& Al-Ofi, S., 2012. Crosswell electromagnetic response in a fractured medium, Geophysics, 77(3), D53-D61, doi:10.1190/geo2011-0227.1.

Dorn, C., Linde, N., Doetsch, J., Borgne, T. L., \& Bour, O., 2012. Fracture imaging within a granitic rock aquifer using multiple-offset single-hole and cross-hole GPR reflection data, Journal of Applied Geophysics, 78, 123-132, doi:10.1016/j.jappgeo.2011.01.010.

Everett, M. E., 2013. Near-Surface Applied Geophysics, Cambridge University Press.

Follin, S., Hartley, L., Rhén, I., Jackson, P., Joyce, S., Roberts, D., \& Swift, B., 2014. A methodology to constrain the parameters of a hydrogeological discrete fracture network model for sparsely fractured crystalline rock, exemplified by data from the proposed highlevel nuclear waste repository site at Forsmark, Sweden, Hydrogeology Journal, 22(2), 313331.

Geiger, S. \& Emmanuel, S., 2010. Non-Fourier thermal transport in fractured geological media, Water Resources Research, 46(7), W07504.

Gisladottir, V. R., Roubinet, D., \& Tartakovsky, D. M., 2016. Particle methods for heat transfer in fractured media, Transport in Porous Media, 115(2), 311-326. 
Greenhalgh, S., Zhou, B., Greenhalgh, M., Marescot, L., \& Wiese, T., 2009. Explicit expressions for the Fréchet derivatives in 3D anisotropic resistivity inversion, Geophysics, 74(3), F31-F43.

Hatton, C., Main, I., \& Meredith, P., 1994. Non-universal scaling of fracture length and opening displacement, Nature, 367(6459), 160.

Herwanger, J., Worthington, M., Lubbe, R., Binley, A., \& Khazanehdari, J., 2004a. A comparison of cross-hole electrical and seismic data in fractured rock, Geophysical Prospecting, 52(2), 109-121.

Herwanger, J. V., Pain, C. C., Binley, A., De Oliveira, C. R. E., \& Worthington, M. H., 2004b. Anisotropic resistivity tomography, Geophysical Journal International, 158(2), 409-425.

Jinsong, S., Benyu, S., \& Naichuan, G., 2009. Anisotropic characteristics of electrical responses of fractured reservoir with multiple sets of fractures, Petroleum Science, 6(2), 127-138.

Kanit, T., Forest, S., Galliet, I., Mounoury, V., \& Jeulin, D., 2003. Determination of the size of the representative volume element for random composites: statistical and numerical approach, International Journal of Solids and Structures, 40(13-14), 3647-3679.

Keller, L. M., 2015. On the representative elementary volumes of clay rocks at the mesoscale, Journal of Geology and Mining Research, 7(6), 58-64.

Klimczak, C., Schultz, R. A., Parashar, R., \& Reeves, D. M., 2010. Cubic law with aperturelength correlation: Implications for network scale fluid flow, Hydrogeology Journal, 18(4), $851-862$.

Kolditz, O. \& Clauser, C., 1998. Numerical simulation of flow and heat transfer in fractured crystalline rocks: Application to the Hot Dry Rock site in Rosemanowes (UK), Geothermics, $\mathbf{2 7}(1), 1-23$.

Lane, J., Haeni, F., \& Watson, W., 1995. Use of a square-array direct-current resistivity method to detect fractures in crystalline bedrock in New Hampshire, Ground Water, 33(3), $476-485$.

Lantuejoul, C., 1991. Ergodicity and integral range, Journal of Microscopy, 161(3), 387-403.

Lapcevic, P., Novakowski, K. S., \& Sudicky, E., 1997. Groundwater flow and solute transport in fractured media, National Water Research Institute.

Li, J. \& Zhang, L., 2010. Geometric parameters and REV of a crack network in soil, Computers and Geotechnics, 37(4), 466-475.

Li, J., Zhang, L., Wang, Y., \& Fredlund, D., 2009. Permeability tensor and representative elementary volume of saturated cracked soil, Canadian Geotechnical Journal, 46(8), 928942.

Li, Y. \& Spitzer, K., 2005. Finite element resistivity modelling for three-dimensional structures with arbitrary anisotropy, Physics of the Earth and Planetary Interiors, 150(1), 15-27. 
Long, J. C. S., Remer, J. S., Wilson, C. R., \& Witherspoon, P. A., 1982. Porous media equivalents for networks of discontinuous fractures, Water Resources Research, 18(3), 645658.

Maillot, J., Davy, P., Le Goc, R., Darcel, C., \& de Dreuzy, J., 2016. Connectivity, permeability, and channeling in randomly distributed and kinematically defined discrete fracture network models, Water Resources Research, 52(11), 8526-8545.

Manna, F., Walton, K. M., Cherry, J. A., \& Parker, B. L., 2017. Mechanisms of recharge in a fractured porous rock aquifer in a semi-arid region, Journal of Hydrology, 555, 869-880.

Marescot, L., Monnet, R., \& Chapellier, D., 2008. Resistivity and induced polarization surveys for slope instability studies in the Swiss Alps, Engineering Geology, 98(1-2), 18-28.

Matheron, G., 1971. The Theory of Regionalized Variables and Its Applications, Centre de Morphologie Mathématique Fontainebleau: Les cahiers du Centre de Morphologie Mathématique de Fontainebleau, École National Supérieure des Mines.

Min, K.-B., Jing, L., \& Stephansson, O., 2004. Determining the equivalent permeability tensor for fractured rock masses using a stochastic REV approach: Method and application to the field data from Sellafield, UK, Hydrogeology Journal, 12(5), 497-510.

NAP, 2015. Characterization, Modeling, Monitoring, and Remediation of Fractured Rock, National Academies of Sciences, Engineering, and Medicine. National Academies Press, Washington, DC.

Neuman, S., 2005. Trends, prospects and challenges in quantifying flow and transport through fractured rocks, Hydrogeology Journal, 13(1), 124-147.

Neuman, S. P., 2008. Multiscale relationships between fracture length, aperture, density and permeability, Geophysical Research Letters, 35(22).

Olson, J. E., 2003. Sublinear scaling of fracture aperture versus length: An exception or the rule?, Journal of Geophysical Research: Solid Earth, 108(B9).

Pain, C. C., Herwanger, J. V., Saunders, J. H., Worthington, M. H., \& de Oliveira, C. R., 2003. Anisotropic resistivity inversion, Inverse Problems, 19(5), 1081.

Pidlisecky, A. \& Knight, R., 2008. FW2_5D: A MATLAB 2.5-D electrical resistivity modeling code, Computers \& Geosciences, 34(12), 1645-1654.

Porsani, J. L., Elis, V. R., \& Hiodo, F. Y., 2005. Geophysical investigations for the characterization of fractured rock aquifers in Itu, SE Brazil, Journal of Applied Geophysics, 57(2), $119-128$.

Pytharouli, S., Lunn, R., Shipton, Z., Kirkpatrick, J., \& do Nascimento, A., 2011. Microseismicity illuminates open fractures in the shallow crust, Geophysical Research Letters, $\mathbf{3 8}(2)$, doi:10.1029/2010GL045875.

Renshaw, C. \& Park, J., 1997. Effect of mechanical interactions on the scaling of fracture length and aperture, Nature, 386(6624), 482. 
Ritzi, R. W. \& Andolsek, R. H., 1992. Relation between anisotropic transmissivity and azimuthal resistivity surveys in shallow, fractured, carbonate flow systems, Ground Water, 30(5), 774-780.

Robert, T., Dassargues, A., Brouyère, S., Kaufmann, O., Hallet, V., \& Nguyen, F., 2011. Assessing the contribution of electrical resistivity tomography (ERT) and self-potential (SP) methods for a water well drilling program in fractured/karstified limestones, Journal of Applied Geophysics, 75(1), 42-53.

Robert, T., Caterina, D., Deceuster, J., Kaufmann, O., \& Nguyen, F., 2012. A salt tracer test monitored with surface ERT to detect preferential flow and transport paths in fractured/karstified limestones, Geophysics, 77(2), B55-B67.

Rotter, B. E., Barry, D. A., Gerhard, J. I., \& Small, J. S., 2008. Modeling U(VI) biomineralization in single- and dual-porosity porous media, Water Resources Research, 44(8).

Roubinet, D. \& Irving, J., 2014. Discrete-dual-porosity model for electric current flow in fractured rock, Journal of Geophysical Research: Solid Earth, 119(2), 767-786.

Roubinet, D., Linde, N., Jougnot, D., \& Irving, J., 2016. Streaming potential modeling in fractured rock: Insights into the identification of hydraulically active fractures, Geophysical Research Letters, 43(10), 4937-4944.

Rücker, C., Günther, T., \& Spitzer, K., 2006. Three-dimensional modelling and inversion of DC resistivity data incorporating topography - I. Modelling, Geophysical Journal International, 166, 495-505.

Schmutz, M., Ghorbani, A., Vaudelet, P., \& Revil, A., 2011. Spectral induced polarization detects cracks and distinguishes between open- and clay-filled fractures, Journal of Environmental and Engineering Geophysics, 16(2), 85-91, doi:10.1190/geo2011-0227.1.

Schön, J. H., 2015. Physical Properties of Rocks: Fundamentals and Principles of Petrophysics, vol. 65, Elsevier.

Sharma, S. \& Baranwal, V., 2005. Delineation of groundwater-bearing fracture zones in a hard rock area integrating very low frequency electromagnetic and resistivity data, Journal of Applied Geophysics, 57(2), 155-166.

Singhal, B. B. S. \& Gupta, R. P., 2010. Applied hydrogeology of fractured rocks, Springer Science \& Business Media.

Skinner, D. \& Heinson, G., 2004. A comparison of electrical and electromagnetic methods for the detection of hydraulic pathways in a fractured rock aquifer, Clare Valley, South Australia, Hydrogeology Journal, 12(5), 576-590.

Skjernaa, L. \& Jørgensen, N. O., 1994. Evaluation of local fracture systems by azimuthal resistivity surveys: Examples from South Norway, Applied Hydrogeology, 2(2), 19-25.

Snow, D. T., 1969. Anisotropic permeability of fractured media, Water Resources Research, 5(6), 1273-1289. 
Steelman, C., Parker, B., \& Kennedy, C., 2015. Evaluating local-scale anisotropy and heterogeneity along a fractured sedimentary bedrock river using EM azimuthal resistivity and ground-penetrating radar, Journal of Applied Geophysics, 116, 156-166.

Steinich, B. \& Marin, L. E., 1996. Hydrogeological investigations in northwestern Yucatan, Mexico, using resistivity surveys, Groundwater, 34(4), 640-646.

Taylor, R. W. \& Fleming, A. H., 1988. Characterizing jointed systems by azimuthal resistivity surveys, Ground Water, 26(4), 464-474.

Telford, W. M., Geldart, L. P., \& Sheriff, R. E., 1990. Applied Geophysics, Cambridge University Press.

Tsoflias, G. P., Halihan, T., \& Sharp, J. M., 2001. Monitoring pumping test response in a fractured aquifer using ground-penetrating radar, Water Resources Research, 37(5), 12211229 .

Van Siclen, C. D., 2002. Equivalent channel network model for permeability and electrical conductivity of fracture networks, Journal of Geophysical Research: Solid Earth, 107(B6).

Vermilye, J. M. \& Scholz, C. H., 1995. Relation between vein length and aperture, Journal of Structural Geology, 17(3), 423-434.

Wang, M. \& Kulatilake, P., 2008. Understanding of hydraulic properties from configurations of stochastically distributed fracture networks, Hydrological Processes, 22(8), 1125-1135.

Willot, F. \& Jeulin, D., 2011. Elastic and electrical behavior of some random multiscale highlycontrasted composites, International Journal for Multiscale Computational Engineering, 9(3).

Wishart, D. N., Slater, L. D., \& Gates, A. E., 2008. Fracture anisotropy characterization in crystalline bedrock using field-scale azimuthal self potential gradient, Journal of Hydrology, 358(1), 35-45.

Yadav, G. \& Singh, S. K., 2007. Integrated resistivity surveys for delineation of fractures for ground water exploration in hard rock areas, Journal of Applied Geophysics, 62(3), $301-312$.

Yeboah-Forson, A. \& Whitman, D., 2014. Electrical resistivity characterization of anisotropy in the Biscayne Aquifer, Groundwater, 52(5), 728-736.

Zhao, J., Al, T., Chapman, S. W., Parker, B. L., Mishkin, K. R., Cutt, D., \& Wilkin, R. T., 2017. Determination of $\mathrm{Cr}(\mathrm{III})$ solids formed by reduction of $\mathrm{Cr}(\mathrm{VI})$ in a contaminated fractured bedrock aquifer: Evidence for natural attenuation of $\mathrm{Cr}(\mathrm{VI})$, Chemical Geology, $474,1-8$. 


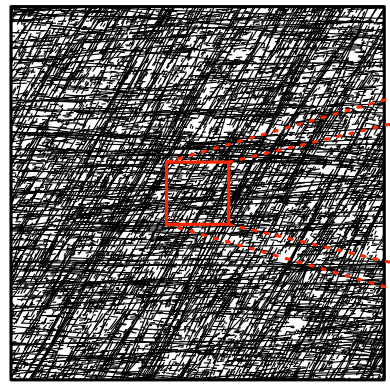

(a)

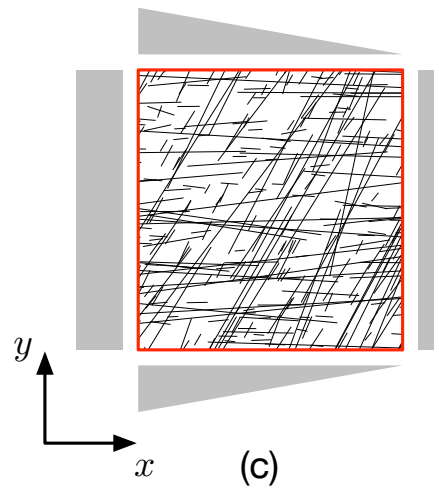

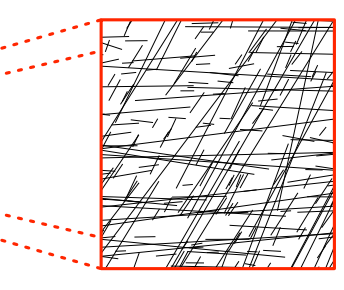

(b)

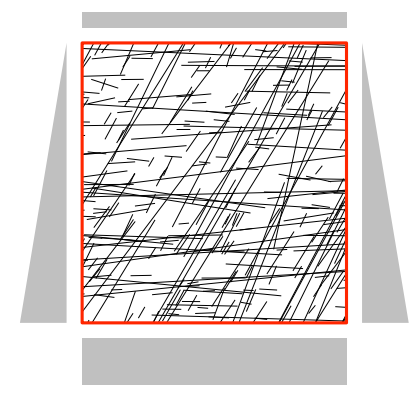

(d)

Figure 1: (a) Example discrete fracture network (DFN) generated over a large-scale square domain. (b) Extraction of central square sub-domain upon which fluid- and electric-current-flow simulations are performed. (c) Dirichlet boundary conditions considered for measuring the conductivity tensor components $C_{x x}$ and $C_{y x}$. (d) Dirichlet boundary conditions considered for measuring components $C_{y y}$ and $C_{x y}$. 

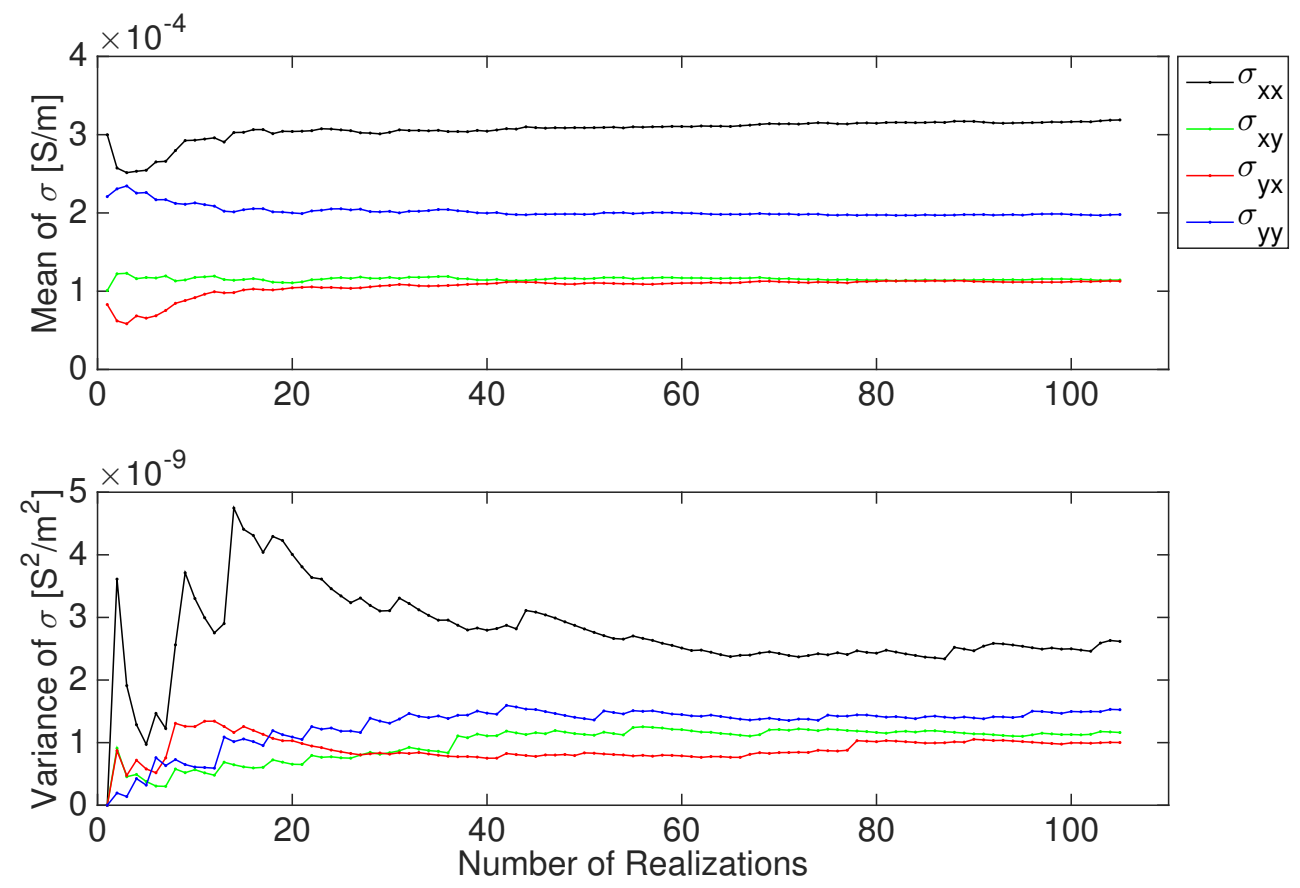

Figure 2: Example showing the calculated mean and variance of the electrical conductivity tensor components $\sigma_{x x}, \sigma_{x y}, \sigma_{y x}$, and $\sigma_{y y}$, plotted as a function of the number of considered fracture-network realizations, for a domain size of $12 \times 12 \mathrm{~m}$. The presented data correspond to Case 12 from Table 1 with $\sigma_{m} / \sigma_{f}=10^{-6}$. See Section 3 for details. 

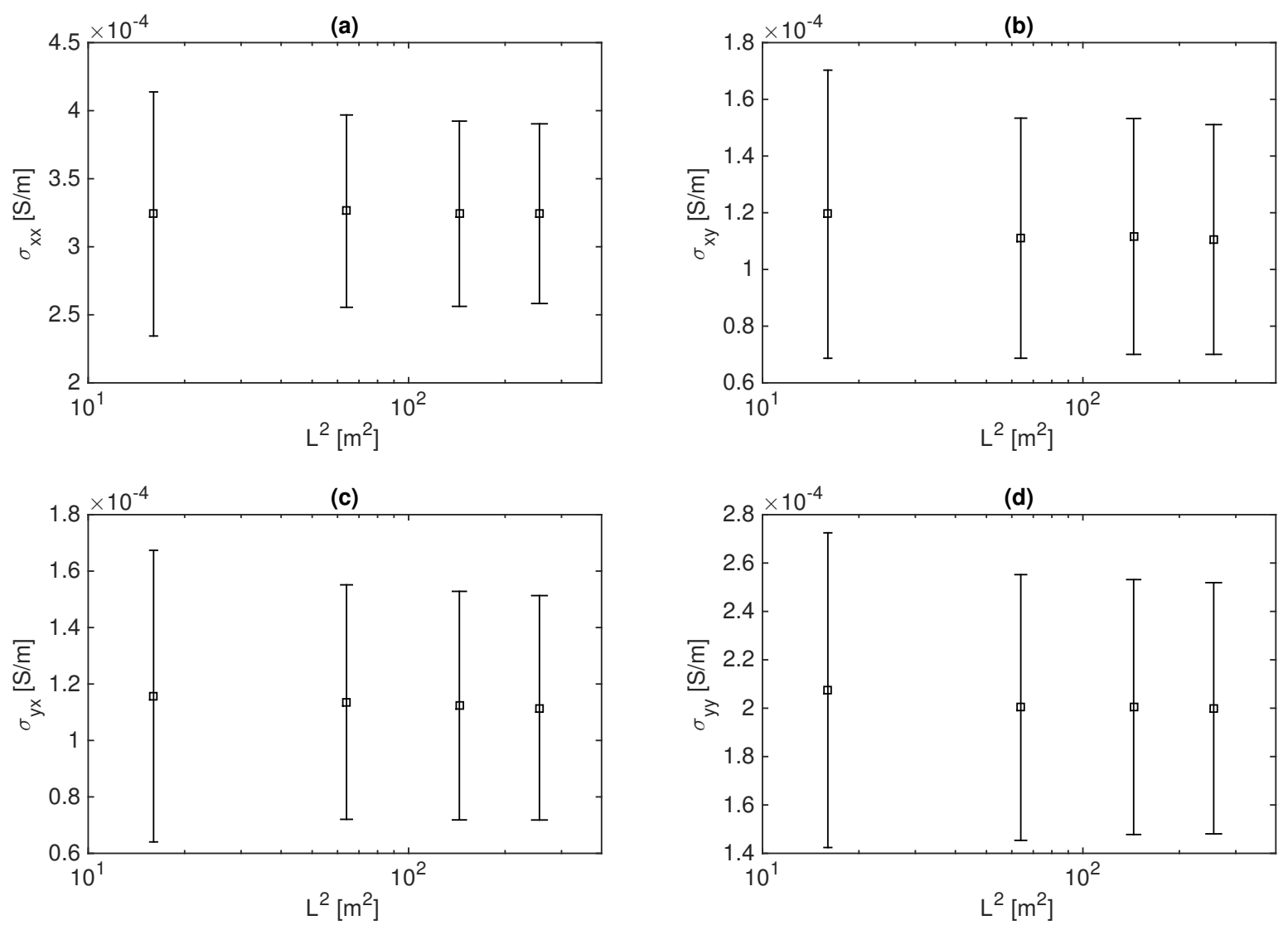

Figure 3: Example showing the stabilized estimates of the mean and standard deviation of the components of the electrical conductivity tensor, plotted as a function of domain area $L^{2}$. The presented data correspond to Case 12 from Table 1 with $\sigma_{m} / \sigma_{f}=10^{-6}$. See Section 3 for details. 


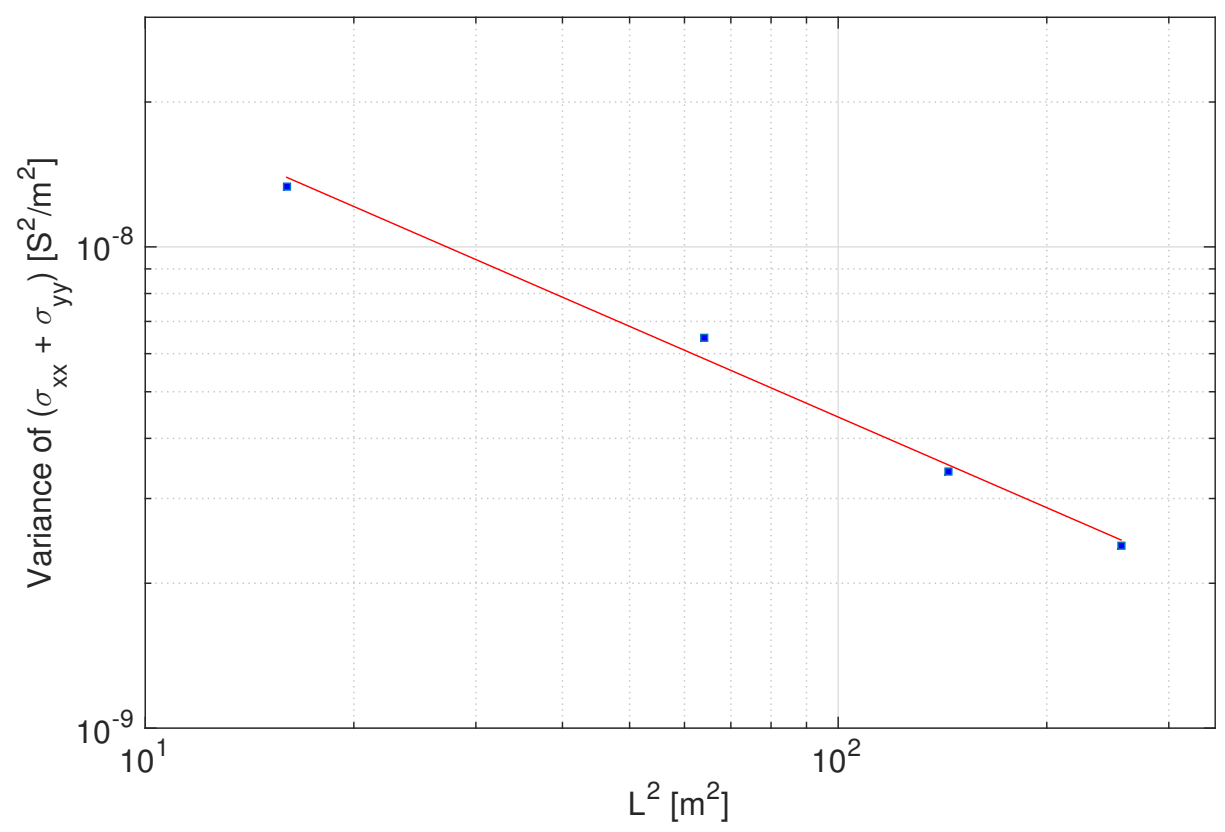

Figure 4: Example showing the variance of $\left(\sigma_{x x}+\sigma_{y y}\right)$ plotted as a function of domain area $L^{2}$. The least-squares best-fitting straight line through the points (red) provides the scaling relationship that is used to determine the REV size. The presented data correspond to Case 12 from Table 1 with $\sigma_{m} / \sigma_{f}=10^{-6}$. See Section 3 for details. 

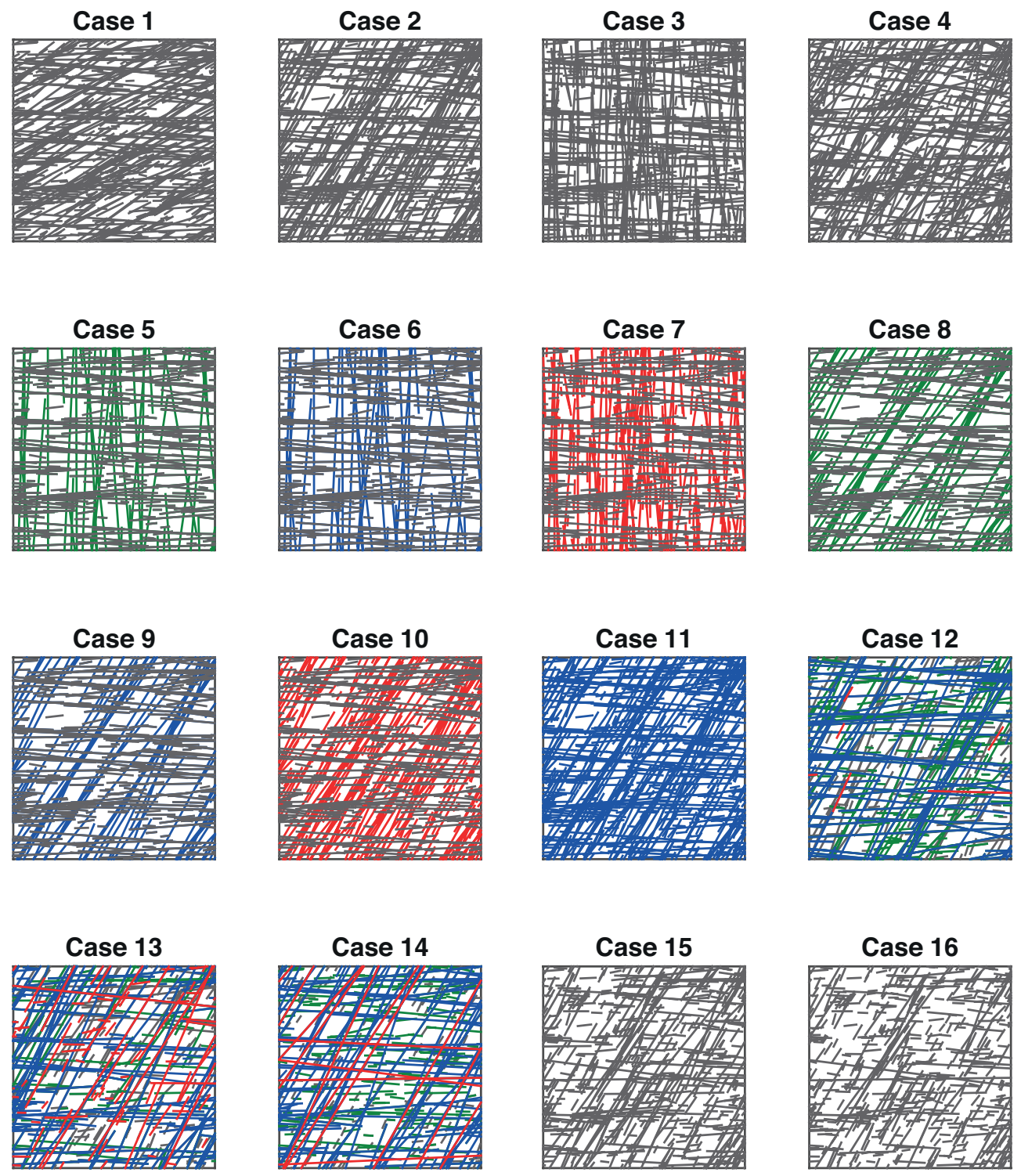

$\mathrm{b} \leq 1 \mathrm{~mm}$; $1<b \leq 1.25 \mathrm{~mm}$ $1.25<b \leq 1.75 \mathrm{~mm}$ $\mathrm{b}>1.75 \mathrm{~mm}$

Figure 5: Example discrete fracture networks corresponding to the different test cases described in Table 1. Each square is $16 \times 16 \mathrm{~m}$ in size. 
(a)

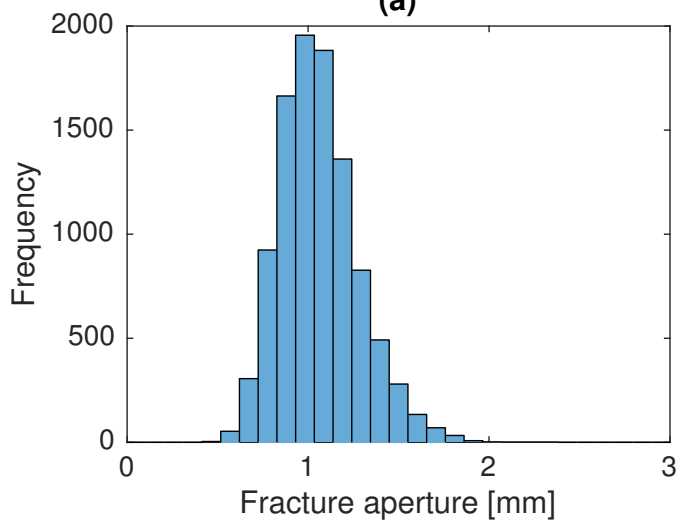

(c)

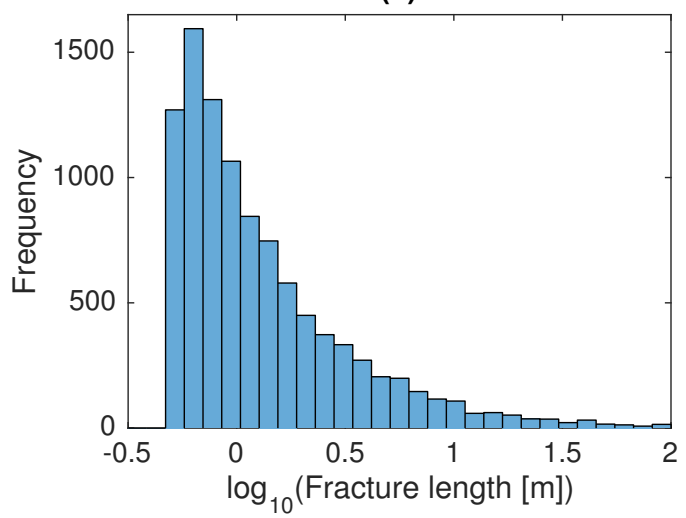

(b)

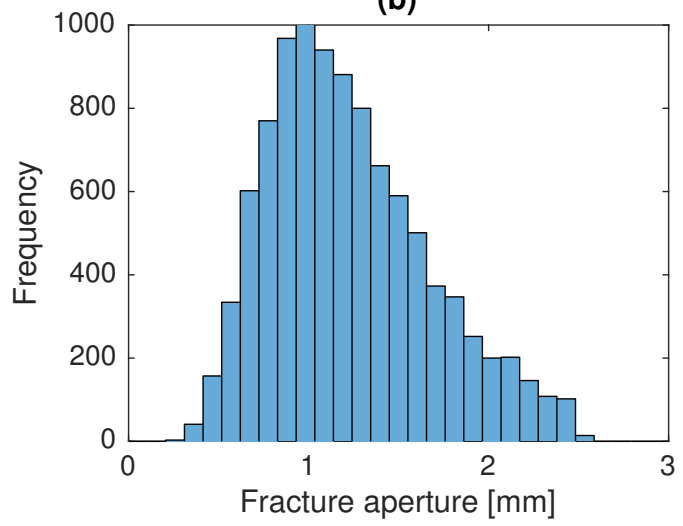

(d)

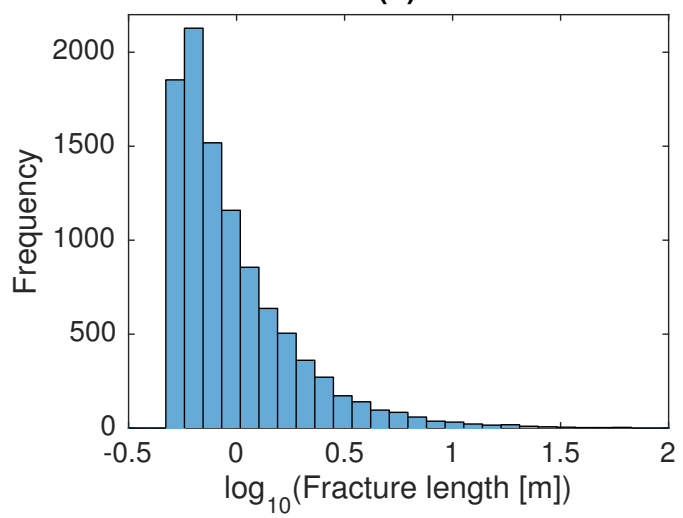

Figure 6: Histograms of the truncated log-normal fracture aperture distribution for (a) $\left(\mu_{\ln b}, \sigma_{\ln b}\right)=(-6.87,0.2)$, and (b) $\left(\mu_{\ln b}, \sigma_{\ln b}\right)=(-6.75,0.4)$, along with histograms of the truncated power-law fracture length distribution for (c) $a=2.0$, and (d) $a=2.5$. 
(a)

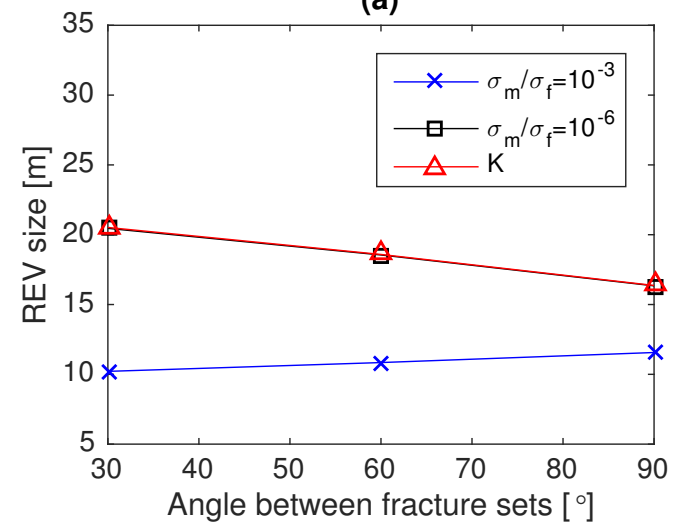

(c)

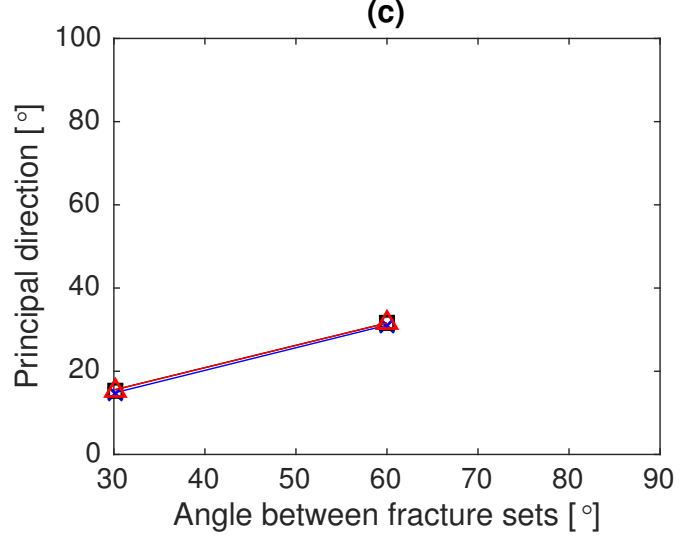

(b)

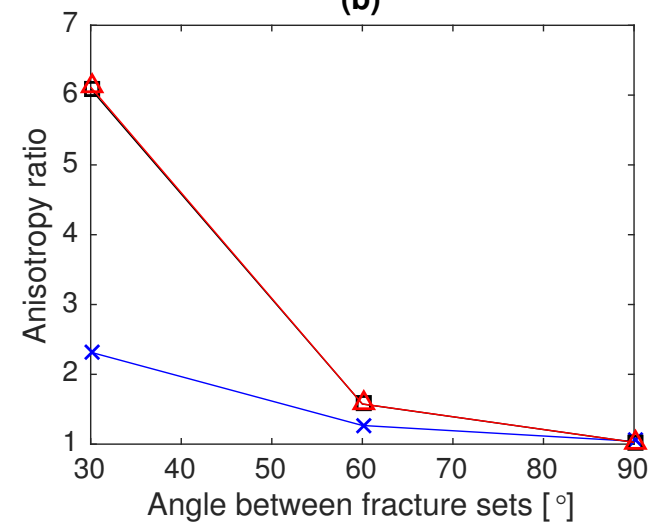

(d)

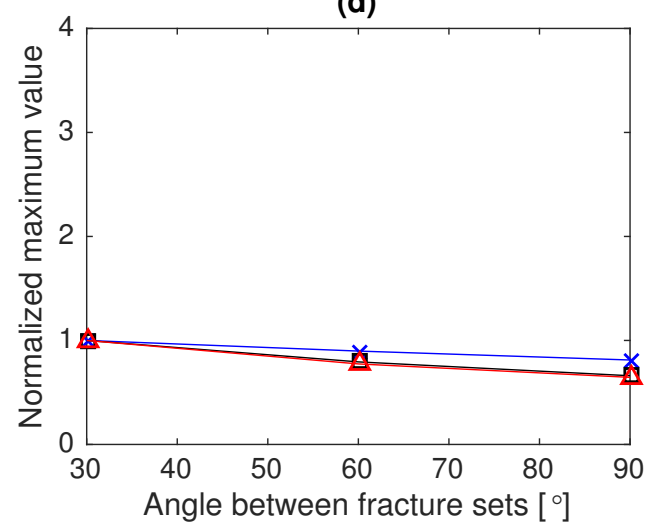

Figure 7: Effect of changing the angle between the two fracture sets on (a) the estimated REV size, (b) the tensor anisotropy ratio, (c) the direction of maximum conductivity; and (d) the maximum conductivity value, normalized between data sets for comparison. Test cases 1, 2, and 3 are considered (Table 1). Note that no principal direction is available when the angle between the fracture sets is $90^{\circ}$ because the system is effectively isotropic (Tables 2 and 3 ). 
(a)

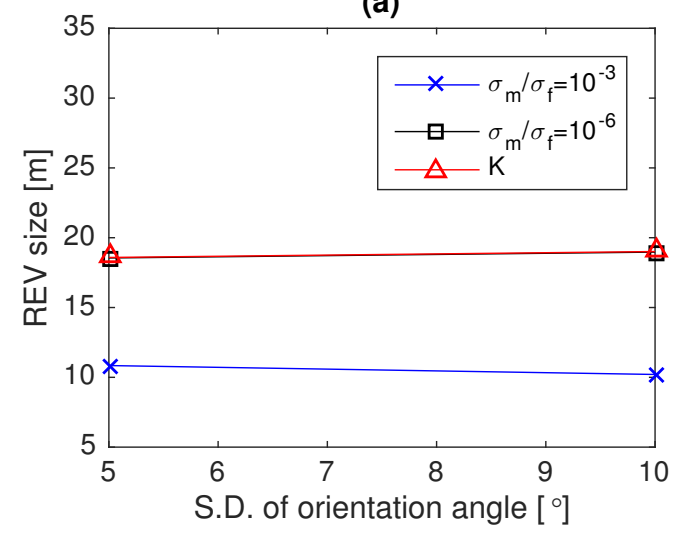

(c)

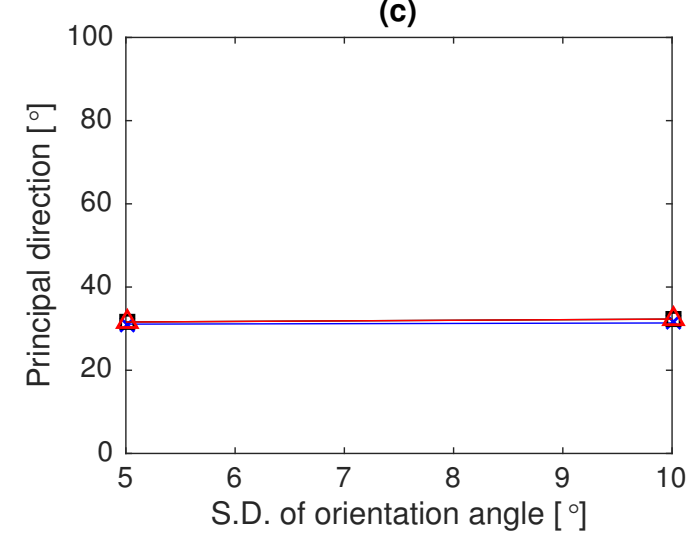

(b)

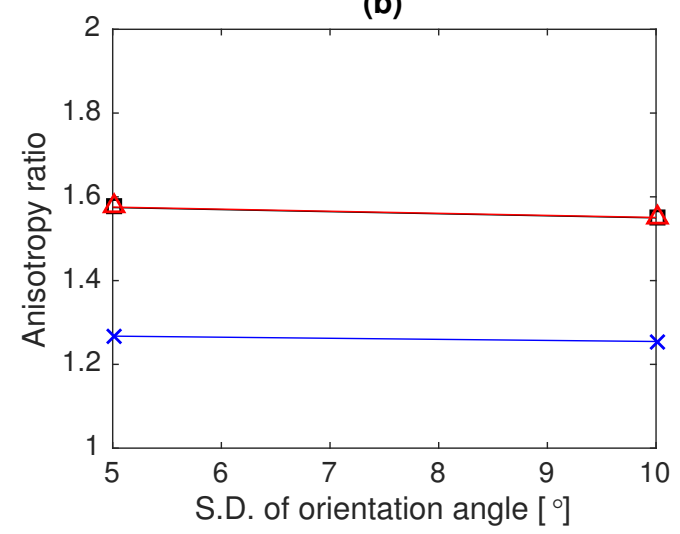

(d)

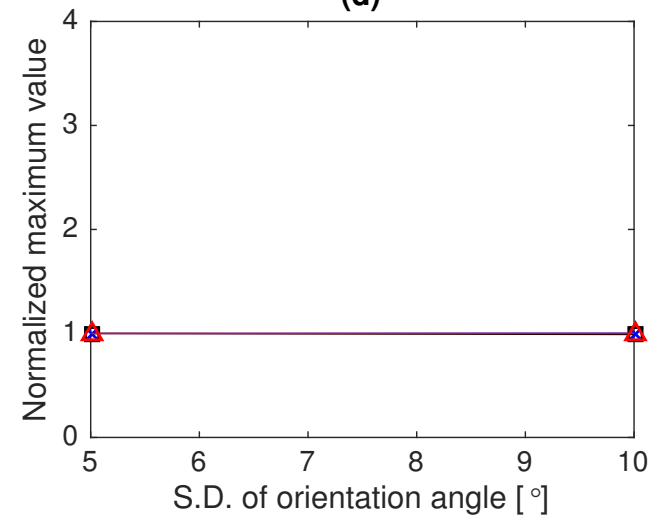

Figure 8: Effect of increasing the standard deviation of the fracture orientation angle on (a) the estimated REV size, (b) the tensor anisotropy ratio, (c) the direction of maximum conductivity; and (d) the maximum conductivity value, normalized between data sets for comparison. Test cases 2 and 4 are considered (Table 1). 
(a)

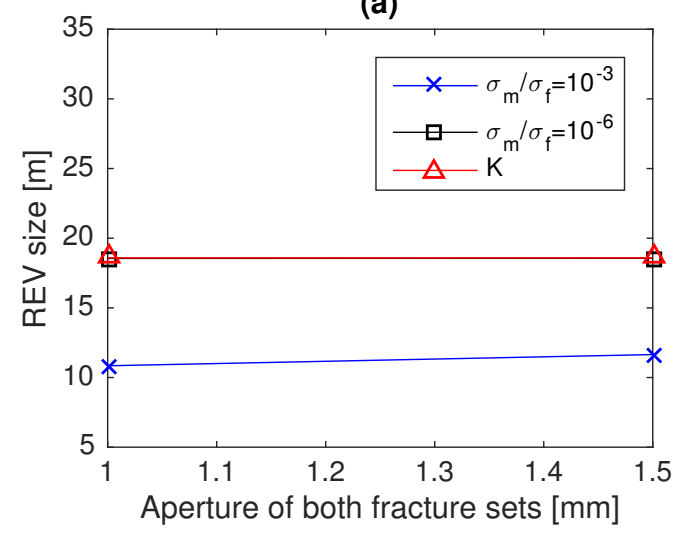

(c)

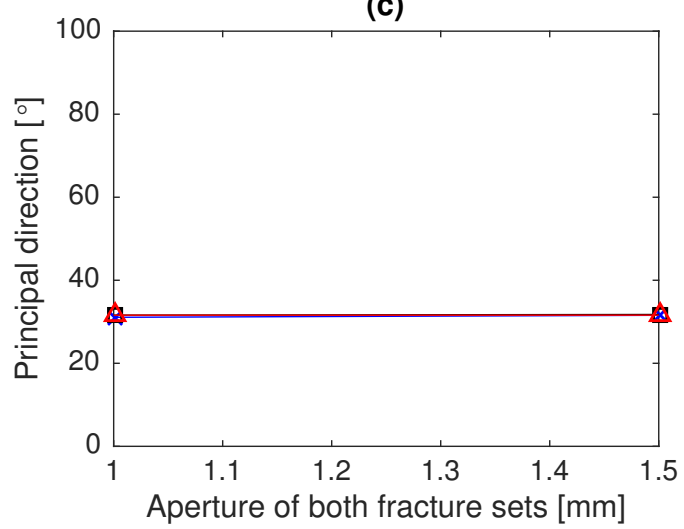

(b)



(d)

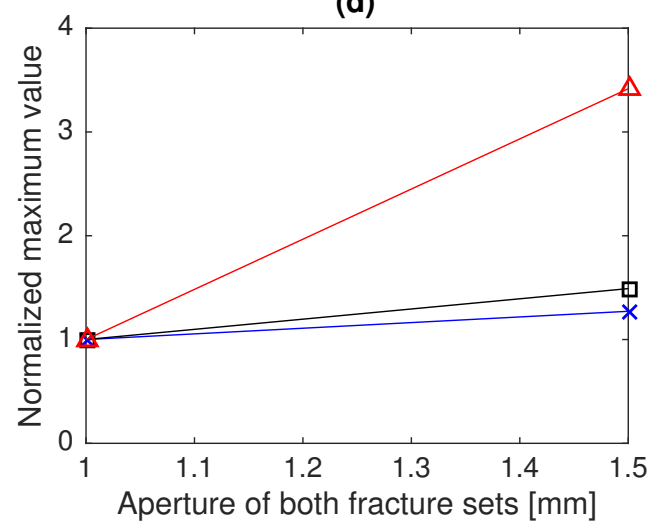

Figure 9: Effect of increasing the aperture of both fracture sets on (a) the estimated REV size, (b) the tensor anisotropy ratio, (c) the direction of maximum conductivity; and (d) the maximum conductivity value, normalized between data sets for comparison. Test cases 2 and 11 are considered (Table 1 ). 
(a)

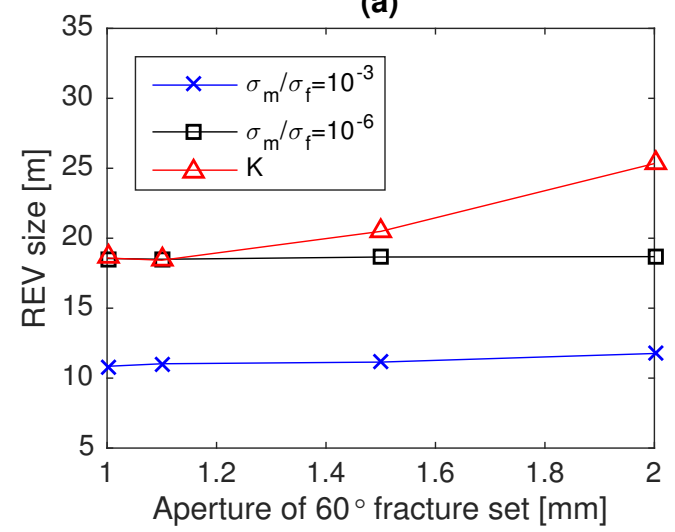

(c)

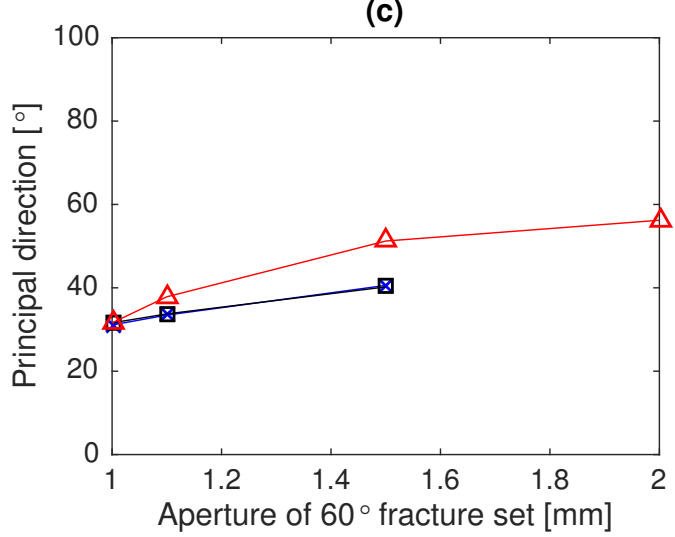

(b)

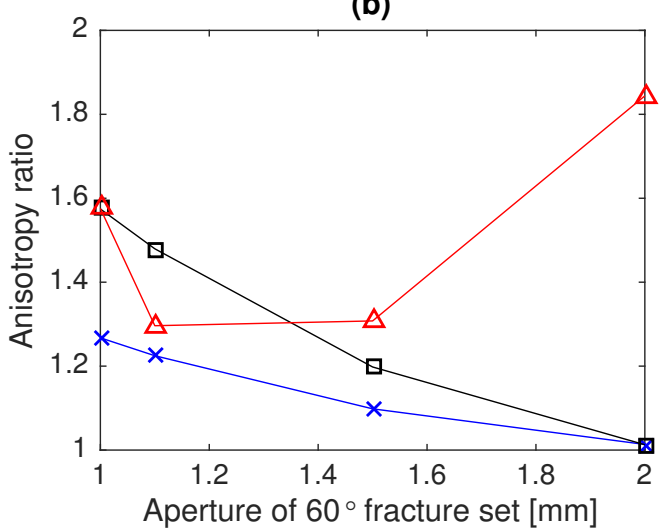

(d)

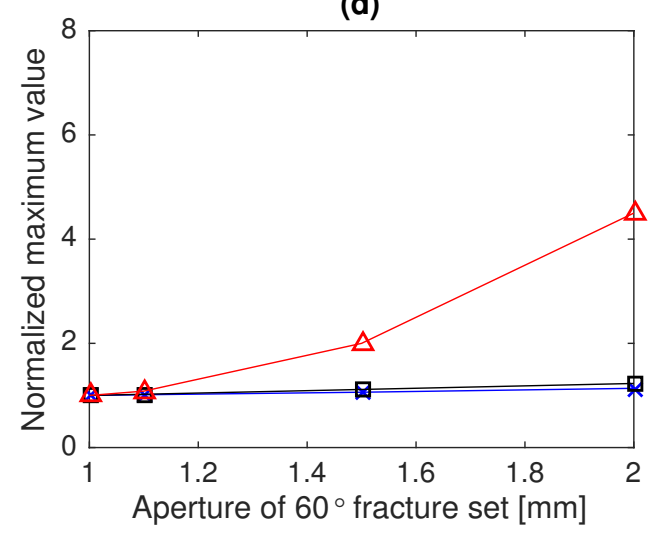

Figure 10: Effect of increasing the aperture of the $60^{\circ}$ fracture set on (a) the estimated REV size, (b) the tensor anisotropy ratio, (c) the direction of maximum conductivity; and (d) the maximum conductivity value, normalized between data sets for comparison. Test cases 2, 8, 9, and 10 are considered (Table 1). Note that no principal direction is available for the electrical conductivity when $b_{2}=2 \mathrm{~mm}$ because the system is effectively isotropic (Tables 2 and 3 ). 
(a)

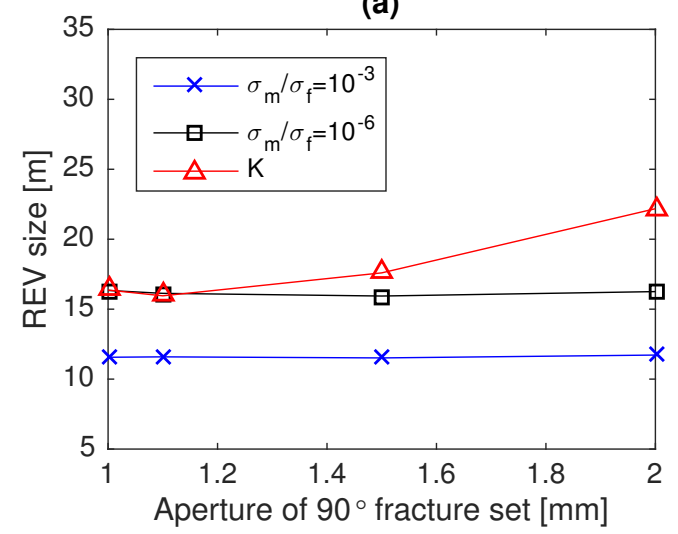

(c)

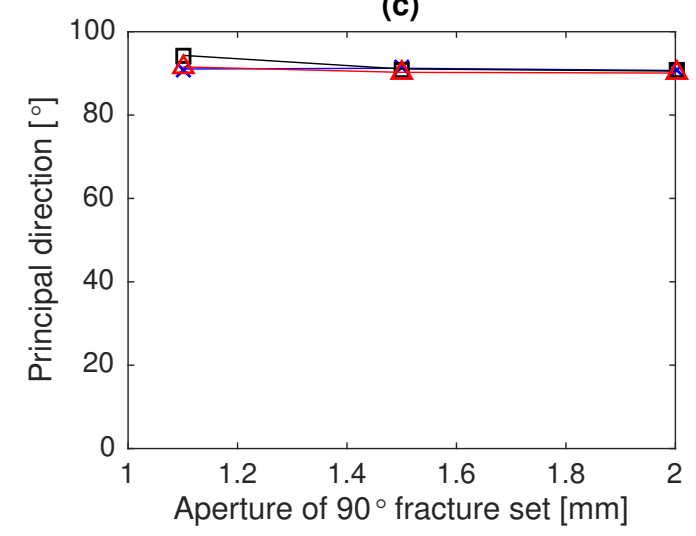

(b)

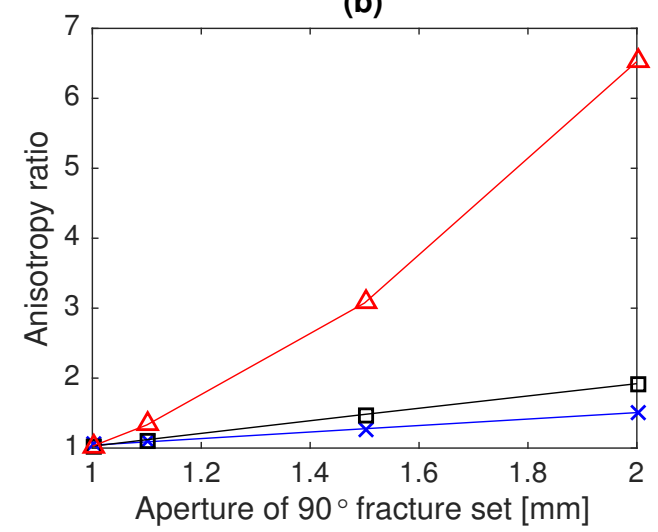

(d)

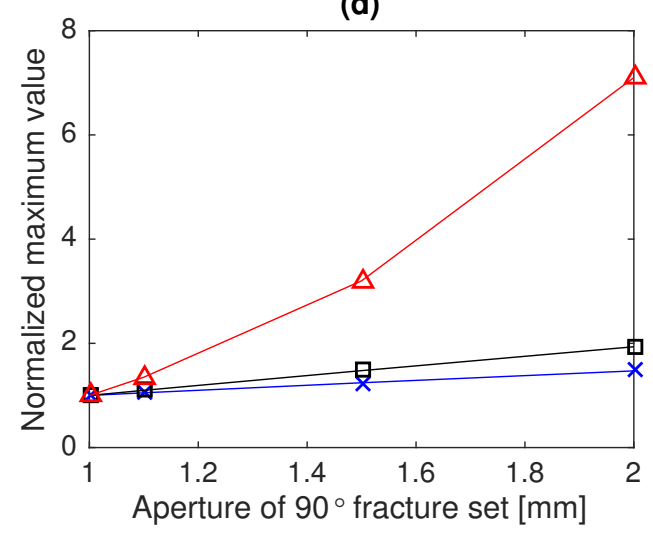

Figure 11: Effect of increasing the aperture of the $90^{\circ}$ fracture set on (a) the estimated REV size, (b) the tensor anisotropy ratio, (c) the direction of maximum conductivity; and (d) the maximum conductivity value, normalized between data sets for comparison. Test cases 3,5 , 6 , and 7 are considered (Table 1 ). 
(a)

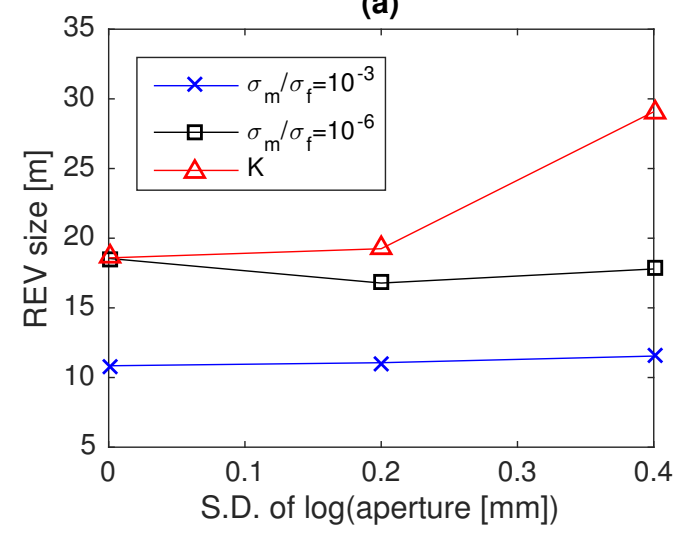

(c)

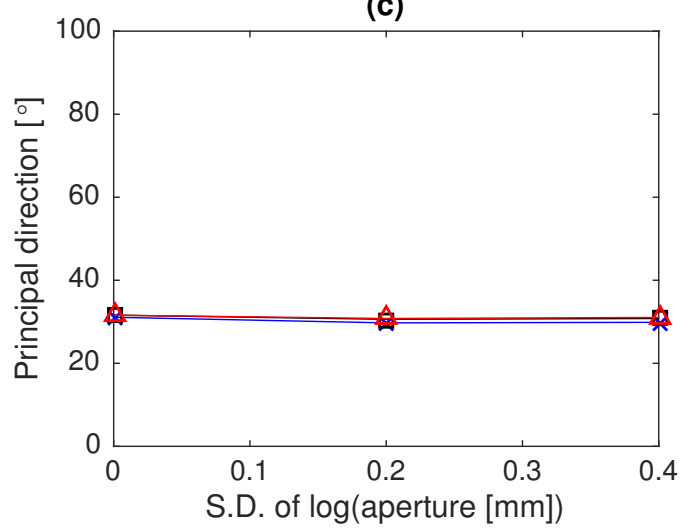

(b)

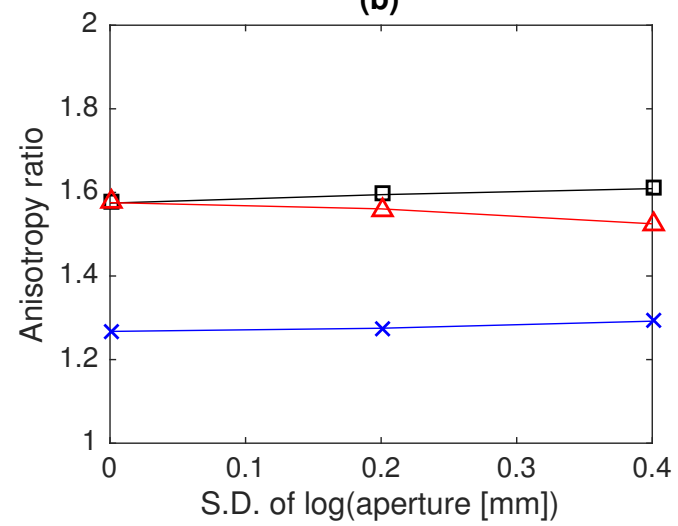

(d)

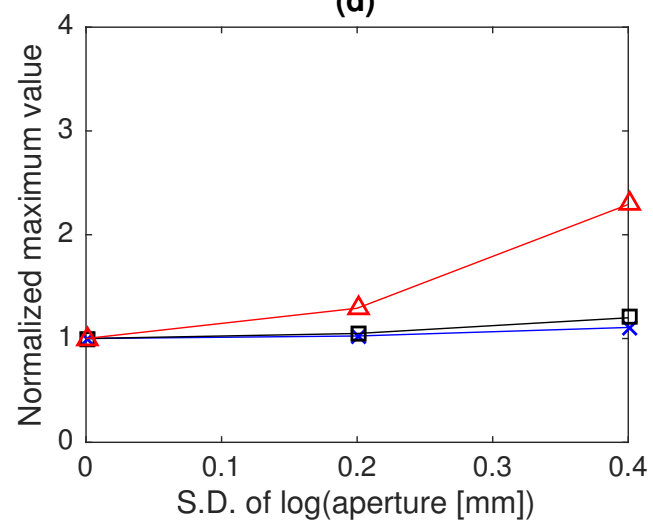

Figure 12: Effect of increasing the fracture-aperture variability on (a) the estimated REV size, (b) the tensor anisotropy ratio, (c) the direction of maximum conductivity; and (d) the maximum conductivity value, normalized between data sets for comparison. Test cases 2, 12, and 13 are considered (Table 1 ). 
(a)

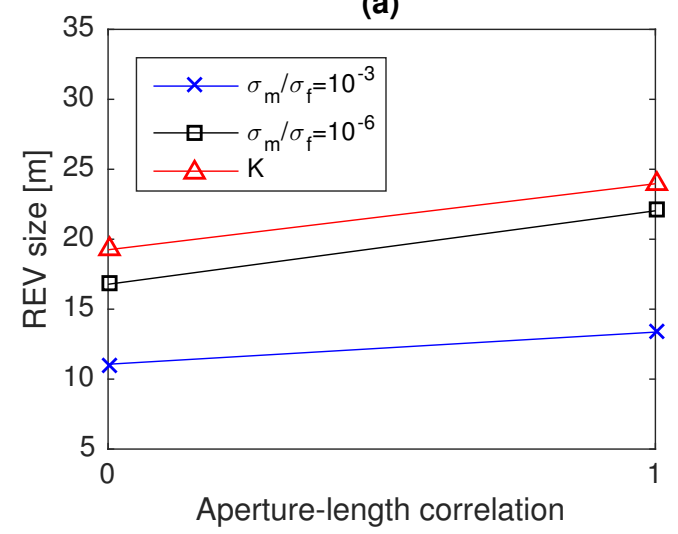

(c)

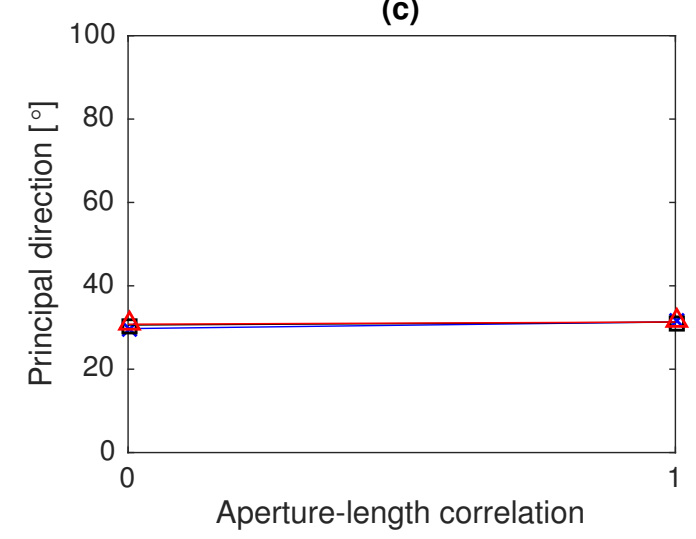

(b)

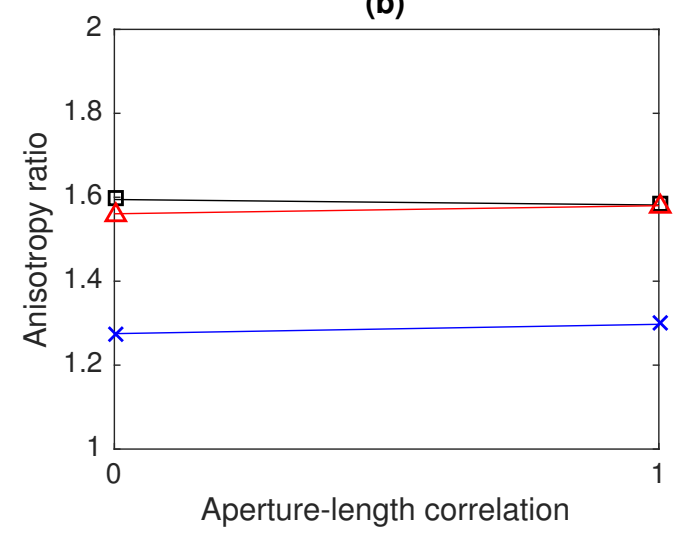

(d)

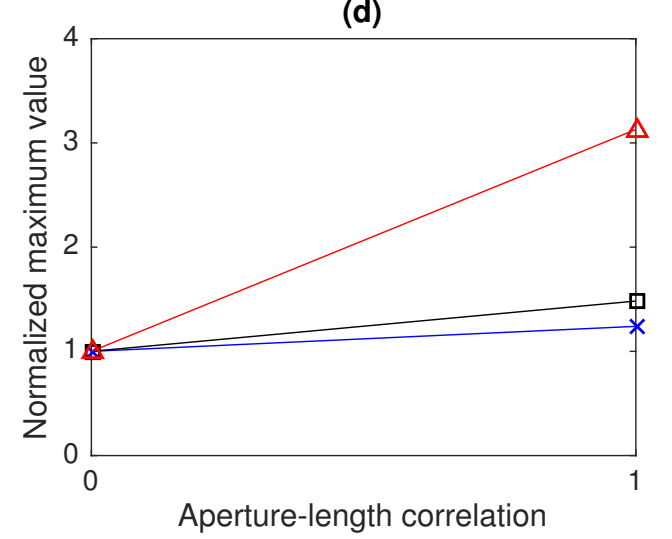

Figure 13: Effect of correlation between aperture and length on (a) the estimated REV size, (b) the tensor anisotropy ratio, (c) the direction of maximum conductivity; and (d) the maximum conductivity value, normalized between data sets for comparison. Test cases 12 and 14 are considered (Table 1). The presence of correlation is indicated on the horizontal axis using a binary variable $(0=$ uncorrelated; $1=$ correlated $)$. 
(a)

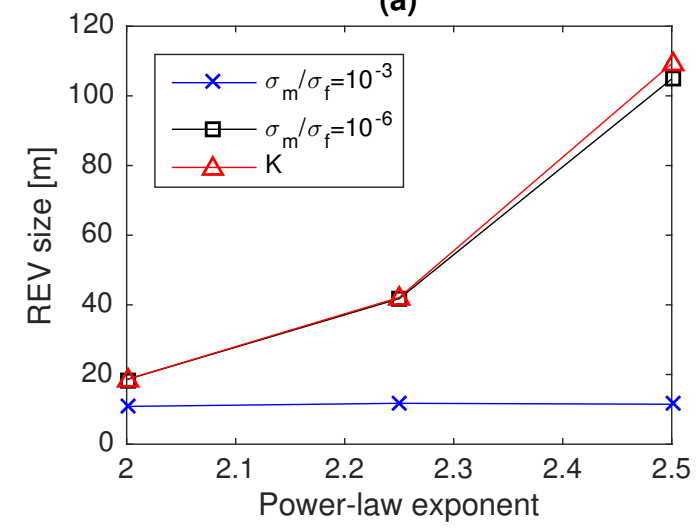

(c)

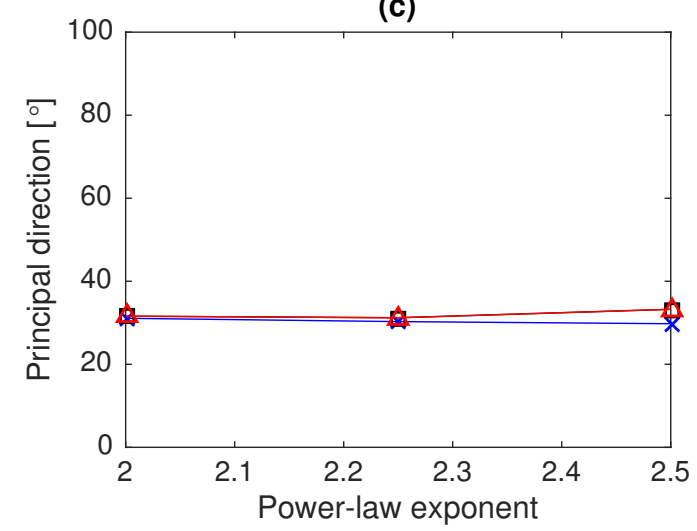

(b)

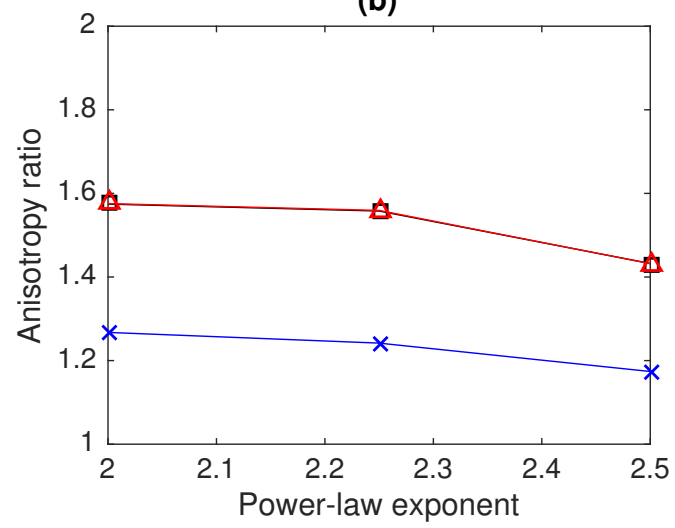

(d)

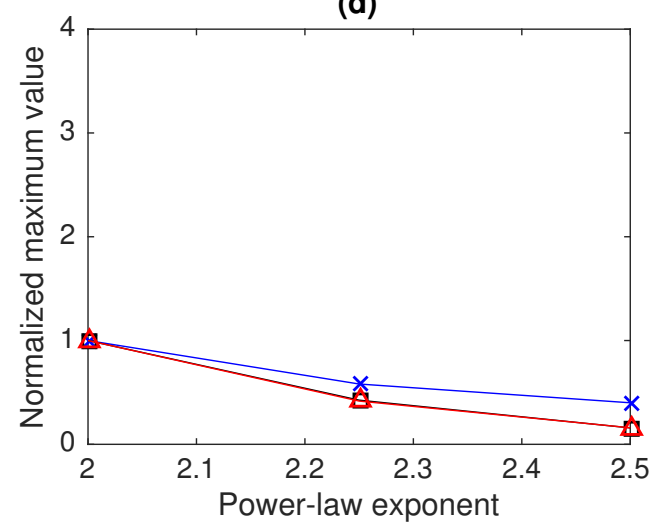

Figure 14: Effect of increasing the fracture-length power-law exponent $a$ on (a) the estimated REV size, (b) the tensor anisotropy ratio, (c) the direction of maximum conductivity; and (d) the maximum conductivity value, normalized between data sets for comparison. Test cases 2,15 , and 16 are considered (Table 1 ). 
Table 1: Values considered in each test case for the orientation angle $(\theta)$, aperture $(b)$, and length power-law exponent $(a)$ for Fracture Sets 1 and 2. $\mathrm{N}\left(\mu_{\theta}, \sigma_{\theta}\right)$ refers to a normal distribution having mean orientation $\mu_{\theta}$ and standard deviation $\sigma_{\theta} \cdot \operatorname{TLN}\left(\mu_{\ln b}, \sigma_{\ln b}\right)$ refers to a truncated $\log$-normal distribution where $\mu_{\ln b}$ and $\sigma_{\ln b}$ are the mean and standard deviation of the natural logarithm of the aperture. The truncation limits for the aperture distribution were set to $b_{\min }=0.1 \mathrm{~mm}$ and $b_{\max }=2.5 \mathrm{~mm}$, whereas the lower limit for the fracture length distribution was set to $\ell_{\min }=0.5 \mathrm{~m}$. See Figure 6 for the corresponding histograms. The right-most column indicates whether fracture aperture and length are correlated.

\begin{tabular}{|c|c|c|c|c|c|c|c|}
\hline Case & $\theta_{1}\left[^{\circ}\right]$ & $b_{1}[\mathrm{~mm}]$ & $a_{1}$ & $\theta_{2}\left[^{\circ}\right]$ & $b_{2}[\mathrm{~mm}]$ & $a_{2}$ & Correlated? \\
\hline 1 & $\mathrm{~N}(0,5)$ & 1.0 & 2.00 & $\mathrm{~N}(30,5)$ & 1.0 & 2.00 & no \\
2 & $\mathrm{~N}(0,5)$ & 1.0 & 2.00 & $\mathrm{~N}(60,5)$ & 1.0 & 2.00 & no \\
3 & $\mathrm{~N}(0,5)$ & 1.0 & 2.00 & $\mathrm{~N}(90,5)$ & 1.0 & 2.00 & no \\
4 & $\mathrm{~N}(0,10)$ & 1.0 & 2.00 & $\mathrm{~N}(60,10)$ & 1.0 & 2.00 & no \\
5 & $\mathrm{~N}(0,5)$ & 1.0 & 2.00 & $\mathrm{~N}(90,5)$ & 1.1 & 2.00 & no \\
6 & $\mathrm{~N}(0,5)$ & 1.0 & 2.00 & $\mathrm{~N}(90,5)$ & 1.5 & 2.00 & no \\
7 & $\mathrm{~N}(0,5)$ & 1.0 & 2.00 & $\mathrm{~N}(90,5)$ & 2.0 & 2.00 & no \\
8 & $\mathrm{~N}(0,5)$ & 1.0 & 2.00 & $\mathrm{~N}(60,5)$ & 1.1 & 2.00 & no \\
9 & $\mathrm{~N}(0,5)$ & 1.0 & 2.00 & $\mathrm{~N}(60,5)$ & 1.5 & 2.00 & no \\
10 & $\mathrm{~N}(0,5)$ & 1.0 & 2.00 & $\mathrm{~N}(60,5)$ & 2.0 & 2.00 & no \\
11 & $\mathrm{~N}(0,5)$ & 1.5 & 2.00 & $\mathrm{~N}(60,5)$ & 1.5 & 2.00 & no \\
12 & $\mathrm{~N}(0,5)$ & $\mathrm{TLN}(-6.87,0.2)$ & 2.00 & $\mathrm{~N}(60,5)$ & $\mathrm{TLN}(-6.87,0.2)$ & 2.00 & no \\
13 & $\mathrm{~N}(0,5)$ & $\mathrm{TLN}(-6.75,0.4)$ & 2.00 & $\mathrm{~N}(60,5)$ & $\mathrm{TLN}(-6.75,0.4)$ & 2.00 & no \\
14 & $\mathrm{~N}(0,5)$ & $\mathrm{TLN}(-6.87,0.2)$ & 2.00 & $\mathrm{~N}(60,5)$ & $\mathrm{TLN}(-6.87,0.2)$ & 2.00 & yes \\
15 & $\mathrm{~N}(0,5)$ & 1.0 & 2.25 & $\mathrm{~N}(60,5)$ & 1.0 & 2.25 & no \\
16 & $\mathrm{~N}(0,5)$ & 1.0 & 2.50 & $\mathrm{~N}(60,5)$ & 1.0 & 2.50 & no \\
\hline
\end{tabular}


Table 2: Estimated REV size and equivalent tensor characteristics for the electrical conductivity $\sigma$ when $\sigma_{m} / \sigma_{f}=10^{-3}$. Parameters $\sigma_{\max }$ and $\sigma_{\min }$ represent the principal values of the conductivity tensor, whereas $\theta_{p}$ is the direction of maximum conductivity. The anisotropy ratio is given by $\sigma_{\max } / \sigma_{\min }$. Note that $\theta_{p}$ is shown only for $\sigma_{\max } / \sigma_{\min } \geq 1.05$.

\begin{tabular}{|c|c|c|c|c|c|}
\hline Case & REV size $[\mathrm{m}]$ & $\sigma_{\max }\left[10^{-4} \mathrm{~S} / \mathrm{m}\right]$ & $\sigma_{\min }\left[10^{-4} \mathrm{~S} / \mathrm{m}\right]$ & $\sigma_{\max } / \sigma_{\min }$ & $\theta_{p}\left[^{\circ}\right]$ \\
\hline 1 & 10.21 & 6.66 & 2.88 & 2.32 & 14.8 \\
2 & 10.84 & 5.98 & 4.72 & 1.27 & 31.1 \\
3 & 11.56 & 5.41 & 5.20 & 1.04 & - \\
4 & 10.20 & 6.00 & 4.78 & 1.25 & 31.4 \\
5 & 11.59 & 5.68 & 5.22 & 1.09 & 91.1 \\
6 & 11.52 & 6.73 & 5.27 & 1.28 & 91.2 \\
7 & 11.72 & 7.96 & 5.28 & 1.52 & 90.7 \\
8 & 11.03 & 6.06 & 4.95 & 1.22 & 33.5 \\
9 & 11.15 & 6.35 & 5.78 & 1.10 & 40.6 \\
10 & 11.76 & 6.80 & 6.71 & 1.01 & - \\
11 & 11.65 & 7.61 & 5.85 & 1.30 & 31.6 \\
12 & 11.06 & 6.13 & 4.81 & 1.28 & 29.7 \\
13 & 11.54 & 6.62 & 5.12 & 1.29 & 29.9 \\
14 & 13.36 & 7.60 & 5.86 & 1.30 & 31.3 \\
15 & 11.74 & 3.48 & 2.80 & 1.24 & 30.3 \\
16 & 11.47 & 2.39 & 2.04 & 1.17 & 29.8 \\
\hline
\end{tabular}


Table 3: Estimated REV size and equivalent tensor characteristics for the electrical conductivity $\sigma$ when $\sigma_{m} / \sigma_{f}=10^{-6}$. Parameters $\sigma_{\max }$ and $\sigma_{\min }$ represent the principal values of the conductivity tensor, whereas $\theta_{p}$ is the direction of maximum conductivity. The anisotropy ratio is given by $\sigma_{\max } / \sigma_{\min }$. Note that $\theta_{p}$ is shown only for $\sigma_{\max } / \sigma_{\min } \geq 1.05$.

\begin{tabular}{|c|c|c|c|c|c|}
\hline Case & REV size $[\mathrm{m}]$ & $\sigma_{\max }\left[10^{-4} \mathrm{~S} / \mathrm{m}\right]$ & $\sigma_{\min }\left[10^{-4} \mathrm{~S} / \mathrm{m}\right]$ & $\sigma_{\max } / \sigma_{\min }$ & $\theta_{p}\left[^{\circ}\right]$ \\
\hline 1 & 20.45 & 3.74 & 0.62 & 6.09 & 15.5 \\
2 & 18.55 & 2.97 & 1.89 & 1.57 & 31.6 \\
3 & 16.34 & 2.47 & 2.41 & 1.03 & - \\
4 & 18.97 & 2.94 & 1.90 & 1.55 & 32.3 \\
5 & 16.13 & 2.71 & 2.42 & 1.12 & 94.3 \\
6 & 15.94 & 3.65 & 2.46 & 1.48 & 91.1 \\
7 & 16.26 & 4.78 & 2.49 & 1.92 & 90.6 \\
8 & 18.50 & 3.04 & 2.05 & 1.48 & 33.7 \\
9 & 18.66 & 3.31 & 2.76 & 1.20 & 40.2 \\
10 & 18.68 & 3.65 & 3.61 & 1.01 & - \\
11 & 18.56 & 4.43 & 2.87 & 1.54 & 31.8 \\
12 & 16.78 & 3.11 & 1.95 & 1.59 & 30.6 \\
13 & 17.79 & 3.56 & 2.21 & 1.61 & 30.8 \\
14 & 22.03 & 4.62 & 2.92 & 1.58 & 31.3 \\
15 & 41.78 & 1.26 & 0.81 & 1.56 & 31.2 \\
16 & 104.87 & 0.47 & 0.33 & 1.43 & 33.2 \\
\hline
\end{tabular}


Table 4: Estimated REV size and equivalent tensor characteristics for the hydraulic conductivity $K$. Parameters $K_{\max }$ and $K_{\min }$ represent the principal values of the conductivity tensor, whereas $\theta_{p}$ is the direction of maximum conductivity. The anisotropy ratio is given by $K_{\max } / K_{\min }$. Note that $\theta_{p}$ is shown only for $K_{\max } / K_{\min } \geq 1.05$.

\begin{tabular}{|c|c|c|c|c|c|}
\hline Case & REV size $[\mathrm{m}]$ & $K_{\max }\left[10^{-3} \mathrm{~m} / \mathrm{s}\right]$ & $K_{\min }\left[10^{-3} \mathrm{~m} / \mathrm{s}\right]$ & $K_{\max } / K_{\min }$ & $\theta_{p}\left[^{\circ}\right]$ \\
\hline 1 & 20.52 & 3.10 & 0.50 & 6.12 & 15.5 \\
2 & 18.59 & 2.40 & 1.50 & 1.58 & 31.6 \\
3 & 16.37 & 2.00 & 2.00 & 1.03 & - \\
4 & 19.01 & 2.40 & 1.50 & 1.55 & 32.3 \\
5 & 15.95 & 2.70 & 2.00 & 1.33 & 91.6 \\
6 & 17.59 & 6.40 & 2.10 & 3.07 & 90.3 \\
7 & 22.18 & 14.20 & 2.20 & 6.53 & 90.1 \\
8 & 18.43 & 2.60 & 2.00 & 1.30 & 37.8 \\
9 & 20.49 & 4.80 & 3.70 & 1.31 & 51.2 \\
10 & 25.34 & 10.80 & 5.90 & 1.84 & 56.2 \\
11 & 18.59 & 8.20 & 5.20 & 1.58 & 31.6 \\
12 & 19.25 & 3.10 & 2.00 & 1.56 & 30.8 \\
13 & 29.07 & 5.50 & 3.60 & 1.52 & 31.0 \\
14 & 23.97 & 9.70 & 6.10 & 1.58 & 31.3 \\
15 & 42.13 & 1.00 & 0.66 & 1.56 & 31.2 \\
16 & 109.26 & 0.38 & 0.27 & 1.43 & 33.2 \\
\hline
\end{tabular}

